\title{
Turbulent Boundary Layer Models: Theory and Applications
}

\author{
José Simão Antunes do Carmo \\ University of Coimbra \\ Portugal
}

\section{Introduction}

Shallow coastal areas are extremely dynamic regions where the fluid motions associated with both surface waves and currents interact with the bottom sediments. The prediction of the wave effects on sediment transport in shallow water conditions and in intermediate depth is still frequently restricted to monochromatic and unidirectional wave models. However, in real shallow water conditions, the nonlinear process of sediment transport responds in a rather different way to the idealized regular wave case. Therefore, in these regions, both the wave non-linearity and the wave-current interaction become important factors to be considered. Forecasts of morphological changes are invariably dependent on the correct prediction of the sand transport rate under the action of waves and currents, which requires accurate estimation of the friction at bed level, considering all resulting complex interactions effects in its entirety. A major consequence of the fluid dynamics resulting from the combined wave and current motions is the response of the movable seabed, which is significantly altered from that expected for a linear superposition of a pure wave motion with a pure current. In recent years, various attempts have been made to improve the state of knowledge of the flow in the bottom boundary layer regarding the wave non-linearity and complex wave-current effects on the sand-transport rate, using theoretical models. The erosion and sediment transport estimation around usual structures in the fluvial and coastal environment, like bridge piers, groynes and breakwaters, are of a major concern for designing these structures and for considering preventive measures. After a brief discussion on turbulence, the following sections present mathematical and numerical approaches of different complexity. Starting by the fundamental equations of the Fluid Mechanics, a complex unresolved formulation without further assumptions is obtained. Afterwards, considering some physical hypotheses, practical models of different complexity are shown, followed by simple parametric approaches and applications.

\section{Turbulence}

Turbulence has been a long standing challenge for human mind. Five centuries after the first studies of Leonardo da Vinci, understanding turbulence continues to attract a great deal of attention. This may be due to its fascinating complexity and ubiquitous presence in a variety of flows in nature and engineering. The first turbulence references by Leonardo da Vinci are based on visual observations. In 1883, Osborne Reynolds introduced the concept of 
averages, which became the base of great theoretical-experimental studies. In $20^{\text {th }}$ century, Taylor by the thirties presented the first statistical theory for isotropic turbulence, Kolmogorov by the year 1941 formulated theoretical developments for local turbulence, Batchelor by the year 1953 distinguish himself for theoretical and experimental studies about free turbulence of waves and jets. Then, much more other studies were presented, mainly about wall turbulence, boundary layer and air models. Several resumes can be found in Monin and Yaglom (1971), Tennekes and Lumley (1972), Launder and Spalding (1972), Hinze (1975), Schiestel (1993), Nezu and Nakagawa (1993), Rodi (1980, 1993), Mohammadi and Pironneau (1994), Lumley (1996), Chen et al. (1996) and Lesieur (1997), among others.

The detailed accurate computation of large scale turbulent flows has become increasingly important and considerable effort has been devoted to the development of models for the simulation of complex turbulent flows in several applications over the last decades. The description of turbulence flows is based on the assumption that instantaneous flow variables satisfy the Navier-Stokes equations, which contain a full description of turbulence, given that they describe the motion of every Newtonian incompressible fluid based on conservation principles without further assumptions. Analysing the applicability of continuum concepts to the description of turbulence, Moulden et al. (1978) conclude that if the Newtonian constitutive relation is valid, then it is plausible to accept that turbulent flows instantaneously satisfy the same dynamical equations as laminar flows. For laminar flows, analytical or numerical solutions can be directly compared to experimental results in some cases. Moser (2006) declared that despite the increasing range of turbulence spatial scales as the Reynolds number increases, in turbulence, the continuum assumption and the Navier-Stokes equations are an increasingly good approximation.

The aforementioned assumption seems to be well supported as DNS "Direct Numerical Simulation", in which all scales of the motion are simulated using solely the Navier-Stokes equations. It is the most natural approach to the numerical simulation of turbulent flows but, since by Kolmogorov's theory, small scales exist down to $\mathrm{O}$. $\left(\operatorname{Re}^{-3 / 4}\right)$, in order to capture them on a mesh, a meshsize $h \approx \operatorname{Re}^{-3 / 4}$ and consequently (in 3D) $N \approx \operatorname{Re}^{9 / 4}$ mesh points are necessary. Thus, it only could be applied for simple and low-Reynolds number turbulent flows (Kaneda \& Ishihara, 2006; McComb, 2011). Even if DNS were feasible for hydraulic practical interest, it is not possible to define, with the precision required by the smallest scales of the motion, proper initial and boundary conditions. This fact is of significant importance due to non-linear character of the advection terms, which results in the production and maintenance of instabilities which in turn excite small scales in the motion. The presence of non-linear terms also precludes the existence, in the most general case, of unique solutions for a given set of initial and boundary conditions. Thus, as a large Reynolds number turbulent flow is inherently unstable, even small boundary perturbations may excite the already existing small scales, with possible corresponding perturbation amplifications. The lack of solution uniqueness and the infeasibility of defining precise initial and boundary conditions combine themselves in a way that the resultant flow appears random in character. Indeed, the uncontrollable nature of the boundary conditions (in terms of wall roughness size and distribution, wall vibration, etc.) forces the analyst to characterize them as "random forcings" which, consequently, produce random responses (Aldama, 1990). The Navier-Stokes equations can then exhibit great sensitivity to initial and boundary conditions leading to unpredictable chaotic behaviour. Although the fundamental laws behind the Navier-Stokes equations are purely deterministic, these 
equations, similar to other simpler deterministic equations, can often behave chaotically under certain conditions. Due to the randomness in turbulent flows, it is hopeless to track instantaneous behaviour. Instead, the goal is to measure this behaviour in the temporal or spatial mean.

Most researchers in the turbulence field accept that instantaneous flow variables satisfy the Navier-Stokes equations as an axiom and use it as the basis for the development of models for numerical simulation. Assuming that details of motion at the level small and intermediate scales, which tend to exhibit high randomness levels and peculiar characteristics such as isotropy, are not required in most applications of interest in engineering and geophysics, the establishment of two approaches, which have the potential for being applied to problems of engineering interest, can be defined. The first approach is based on the use of filters for the flow variables of interest, Large Eddy Simulation (LES). The second one relies on the use of statistical averages on the same variables, Reynolds-averaged Navier-Stokes equations. Although the former is formally superior to the latter, its use implies paying a computational price which is too high for applications of practical interest. LES requires less computational effort than direct numerical simulation (DNS), but more effort than those methods that solve the Reynoldsaveraged Navier-Stokes equations (RANS). These equations, derived by Osborne Reynolds in 1985, describe the dynamics of the "mean flow" in terms of a time average, and later defined as average in the probability space "ensemble average". The Reynolds stresses produced by advection terms, which are second order correlations in statistical terms, are determined by exact transport equations for the Reynolds stresses derived from the Navier-Stokes equations. However, third-order correlations appear in such expressions and four-order correlations will appear in the exact transport equations for the third-order correlations. This is called the problem of closure of the statistical treatment. The approach of neglecting correlations of higher order has proved to be unsuccessful because the turbulent flows are not completely random. Experimental investigations have made it possible to identify, through the use of conditional sampling techniques, "coherent structures" such as shear layers imbedded in turbulent flows, and that the degree of coherence is scale dependent. In the solution of complicated sets of nonlinear partial differential equations, the interaction between physics and numerical approach is very strong, and the use of second approach in question makes it possible to have a better understanding of that interaction and, as a consequence, to control it. Four main approaches have been followed to find ways to close the Reynolds equations by introducing hypotheses based on physical insight and observational evidence: 1transport; 2- mean velocity field; 3- turbulent field, and 4- invariant models. The resulting model equations contain a number of empirical constants which, in general, increase with their complexity. These models have the base on important concepts and hypotheses as the eddy viscosity concept by Boussinesq, in 1877, Prandtl's mixing length concept, in 1925, Kolmogorov's isotropic dissipation assumption, in 1941, and Rotta's energy redistribution hypothesis, in 1951 (Monin \& Yaglom, 1971; Rodi, 1984).

\section{Governing equations}

The fundamental equations of the Fluid Mechanics applied to a three-dimensional flow of an incompressible and viscous fluid, with sediment in suspension, are written: 

a) $\frac{\partial u_{i}}{\partial x_{i}}=0$
b) $\frac{\partial u_{i}}{\partial t}+u_{j} \frac{\partial u_{i}}{\partial x_{j}}=-\frac{1}{\rho_{0}} \frac{\partial p}{\partial x_{i}}+v_{l} \frac{\partial^{2} u_{i}}{\partial x_{j}^{2}}+g_{i} \frac{\rho-\rho_{0}}{\rho_{0}}$
c) $\frac{\partial C}{\partial t}+\left(u_{i}+w_{s_{i}}\right) \frac{\partial C}{\partial x_{i}}=\gamma_{m} \frac{\partial^{2} C}{\partial x_{i}^{2}}$
d) $\rho=\rho_{s} C+(1-C) \rho_{0}$

where $u$ is the instantaneous velocity of the flow; $C$ is the volumetric concentration of the sediment; $p$ is the pressure; $v_{l}$ is the kinematic viscosity; $g_{i}$ is the acceleration due to gravity; $\rho$ is the density; $\rho_{0}$ is the density of the fluid; $\rho_{s}$ is the density of the sediment; $w_{s}$ is the sediment settling velocity, and $\gamma_{m}$ is the molecular diffusivity.

\subsection{Turbulence closure model with sediment in suspension}

Following the classical Osborne Reynolds procedure, and assuming that the fluid is in a randomly unsteady turbulent state and applying time averaging to the basic equations of motion, the fundamental equations of incompressible turbulent motion are obtained. These are known as the Reynolds equations, and involve both mean and fluctuating quantities the turbulent inertia tensor components. We consider only incompressible turbulent flow with constant transport properties but with possible significant fluctuations in velocity, pressure, and concentration, i.e.:

$$
u_{i}=\bar{u}_{i}+u_{i}^{\prime} ; p_{i}=\bar{p}_{i}+p_{i}^{\prime} ; C_{i}=\bar{C}_{i}+C_{i}^{\prime}
$$

Substituting these functions into the basic equations (1), and taking the time average of each entire equation, we obtain (2) (Rodi, 1984):
a) $\frac{\partial \bar{u}_{i}}{\partial x_{i}}=0$
b) $\frac{\partial \bar{u}_{i}}{\partial t}+\bar{u}_{j} \frac{\partial \bar{u}_{i}}{\partial x_{j}}=-\frac{1}{\rho_{0}} \frac{\partial \bar{p}}{\partial x_{i}}+\frac{\partial}{\partial x_{j}}\left[v_{l} \frac{\partial \bar{u}_{i}}{\partial x_{j}}-\overline{u_{i}^{\prime} u_{j}^{\prime}}\right]+g_{i} \frac{\bar{\rho}-\rho_{0}}{\rho_{0}}$
c) $\frac{\partial \overline{\mathrm{C}}}{\partial t}+\left(\bar{u}_{i}+w_{s_{i}}\right) \frac{\partial \overline{\mathrm{C}}}{\partial x_{i}}=\frac{\partial}{\partial x_{i}}\left[\gamma_{m} \frac{\partial \overline{\mathrm{C}}}{\partial x_{i}}-\overline{u_{i}^{\prime} \rho^{\prime}}\right]$
d) $\bar{\rho}=\rho_{s} \bar{C}+(1-\bar{C}) \rho_{0}$

where $-\overline{u_{i}^{\prime} u_{j}^{\prime}}$ are the tensor components of the Reynolds stresses, and $\overline{-u_{i}^{\prime} \rho^{\prime}}$ are the tensor components of density-velocity correlations. Thus the mean momentum equation and the equation for the concentration are complicated by new terms involving the turbulent inertia tensor $u_{i}^{\prime} u_{j}^{\prime}$ and density fluctuations $u_{i}^{\prime} \rho^{\prime}$. The new terms are never negligible in any turbulent flow with sediment in suspension, and can be defined only through knowledge of the detailed turbulent structure, which is, in its turn, unavailable. These turbulent quantities are related not only to the fluid physical properties but also to local flow conditions. As no physical laws are available, most attempts have been made to resolve this dilemma. Many 
attempts have been made to add turbulence conservation relations to the time-averaged equations above.

\subsection{Boussinesq hypothesis (first order turbulence closure model)}

According to the Boussinesq hypothesis, the turbulent shear stresses $\overline{u_{i}^{\prime} u_{j}^{\prime}}$ are modelled in terms of the gradients of the mean flow velocities through (3),

$$
\overline{-u_{i}^{\prime} u_{j}^{\prime}}=v_{t}\left[\frac{\partial \bar{u}_{i}}{\partial x_{j}}+\frac{\partial \bar{u}_{j}}{\partial x_{i}}\right]-\frac{2}{3} \delta_{i j} K ;-\overline{u_{i}^{\prime} \rho^{\prime}}=\gamma_{t} \frac{\partial \bar{\rho}}{\partial x_{i}} ; i, j=1,2,3
$$

where $K=u_{i}^{\prime} u_{j}^{\prime} / 2=\left(\overline{u_{1}^{\prime 2}}+\overline{u_{2}^{\prime 2}}+\overline{u_{3}^{\prime 2}}\right) / 2$ is the turbulent kinetic energy, per mass unit; $v_{t}$ is the turbulent viscosity, and $\gamma_{t}$ is the turbulent diffusivity. In contrast to the molecular viscosity $v_{l}$, the turbulent viscosity $v_{t}$ is not a fluid property, but depends strongly on the state of the turbulence and may vary considerably over the flow field. A turbulence model thus usually has the task of determining the distribution of $v_{t}$ over the flow field, by relating the turbulence correlations to the averaged dependent variables. As a first order turbulence closure, the turbulent viscosity $v_{t}$ is obtained through the mixing-length theory of Prandtl (1925), who, by analogy with kinetic theory, proposed that each turbulent fluctuation could be related to a length $l_{m}$ scale and a velocity gradient,

$$
v_{t}=l_{m}^{2}\left|\frac{\partial u_{i}}{\partial z}\right|=l_{m}^{2} \sqrt{\left(\frac{\partial u}{\partial z}\right)^{2}+\left(\frac{\partial v}{\partial z}\right)^{2}}
$$

For the $l_{m}$ scale different relations have been proposed. We suggest $l_{m}=k z \sqrt{1-z / z_{\delta}}$, where $k \approx 0.4$ is the von Kármán constant and $z_{\delta}$ is the boundary layer thickness.

\subsection{Second order turbulence closure model}

A derivation of the turbulent shear stresses, where $i \neq j$, involves subtracting the above time-averaged equation (2-b) from its instantaneous value (1-b), for both the $x_{i}$ and $x_{j}$ directions. The $i$ th result is then multiplied by $u_{j}^{\prime}$ and added to the $j$ th result multiplied by $u_{i}^{\prime}$. This relation is then time-averaged to yield the following Reynolds stress equation $\overline{u_{i}^{\prime} u_{j}^{\prime}}$ :

$$
\begin{aligned}
\frac{\partial}{\partial t}\left(\overline{u_{i}^{\prime} u_{j}^{\prime}}\right) & +\bar{u}_{k} \frac{\partial}{\partial x_{k}}\left(\overline{u_{i}^{\prime} u_{j}^{\prime}}\right)=-\overline{u_{i}^{\prime} u_{k}^{\prime}} \frac{\partial \bar{u}_{j}}{\partial x_{k}}-\overline{u_{j}^{\prime} u_{k}^{\prime}} \frac{\partial \bar{u}_{i}}{\partial x_{k}}+\frac{1}{\rho_{0}}\left(\overline{g_{i} u_{j}^{\prime} \rho^{\prime}}+\overline{g_{j} u_{i}^{\prime} \rho}\right) \\
& -\frac{\partial}{\partial x_{k}}\left(\overline{u_{i}^{\prime} u_{j}^{\prime} u_{k}^{\prime}}\right)-\frac{1}{\rho_{0}}\left(\overline{u_{i}^{\prime} \frac{\partial p}{\partial x_{j}}}+\overline{u_{j}^{\prime} \frac{\partial p}{\partial x_{i}}}\right)+v_{l} \frac{\partial^{2}}{\partial x_{k}^{2}}\left(\overline{u_{i}^{\prime} u_{j}^{\prime}}\right)-2 v_{l} \frac{\partial u_{i}^{\prime}}{\partial x_{k}} \frac{\partial u_{j}^{\prime}}{\partial x_{k}}
\end{aligned}
$$

In equation (5), the three terms of different nature $-\frac{\partial}{\partial x_{k}}\left(\overline{u_{i}^{\prime} u_{j}^{\prime} u_{k}^{\prime}}\right),-\frac{1}{\rho_{0}}\left(\overline{u_{i}^{\prime} \frac{\partial p}{\partial x_{j}}}+\overline{u_{j}^{\prime} \frac{\partial p}{\partial x_{i}}}\right)$ and $v_{l} \frac{\partial^{2}}{\partial x_{k}^{2}}\left(\overline{u_{i}^{\prime} u_{j}^{\prime}}\right)-2 v_{l} \overline{\frac{\partial u_{i}^{\prime}}{\partial x_{k}} \frac{\partial u_{j}^{\prime}}{\partial x_{k}}}$ are to be either neglected or related to other variables. Let us consider these terms in some detail. 
- The third-order velocity correlations $-\frac{\partial}{\partial x_{k}}\left(\overline{u_{i}^{\prime} u_{j}^{\prime} u_{k}^{\prime}}\right)$ express the process as the Reynolds stresses are conservatively transmitted from one region of the flow to another; they are usually obtained through $C_{t} \frac{\partial}{\partial x_{k}}\left[\bar{q} L \frac{\partial}{\partial x_{k}}\left(\overline{u_{i}^{\prime} u_{j}^{\prime}}\right)\right]$ (Lewellen, 1977), where $\bar{q}$ is the root-mean-square value of the total velocity fluctuation, $L$ is the macroscale of the eddies, and $C_{t}$ is a constant;

- The pressure-velocity correlations $-\frac{1}{\rho_{0}}\left(\overline{u_{i}^{\prime} \frac{\partial p}{\partial x_{j}}}+\overline{u_{j}^{\prime} \frac{\partial p}{\partial x_{i}}}\right)$, which redistribute to the mean flow the turbulent energy produced, are commonly approximated by $-C_{p} \frac{\sqrt{\overline{q^{2}}}}{L}\left(\overline{u_{i}^{\prime} u_{j}^{\prime}}-\frac{1}{3} \delta_{i, j} \overline{q^{2}}\right)$, where $\delta_{i, j}$ is the Kronecker symbol and $C_{p}$ is a constant;

- The dissipation terms $v_{l} \frac{\partial^{2}}{\partial x_{k}^{2}}\left(\overline{u_{i}^{\prime} u_{j}^{\prime}}\right)$ and $-2 v_{l} \frac{\partial u_{i}^{\prime}}{\partial x_{k}} \frac{\partial u_{j}^{\prime}}{\partial x_{k}}$, which represent the destruction of the mean flow energy by viscous effects, are jointly modelled through $-C_{v} \frac{1}{L} \delta_{i, j}\left(\overline{q^{2}}\right)^{3 / 2}$, where $C_{v}$ is a constant. Inserting these approximations in (5), the following equation (6) for the $\overline{u_{i}^{\prime} u_{j}^{\prime}}$ correlations is obtained:

$$
\begin{aligned}
\frac{\partial}{\partial t}\left(\overline{u_{i}^{\prime} u_{j}^{\prime}}\right) & +\bar{u}_{k} \frac{\partial}{\partial x_{k}}\left(\overline{u_{i}^{\prime} u_{j}^{\prime}}\right)=-\overline{u_{i}^{\prime} u_{k}^{\prime}} \frac{\partial \bar{u}_{j}}{\partial x_{k}}-\overline{u_{j}^{\prime} u_{k}^{\prime}} \frac{\partial \bar{u}_{i}}{\partial x_{k}}+\frac{1}{\rho_{0}}\left(\overline{g_{i} u_{j}^{\prime} \rho^{\prime}}+\overline{g_{j} u_{i}^{\prime} \rho^{\prime}}\right) \\
& +C_{t} \frac{\partial}{\partial x_{k}}\left[\bar{q} L \frac{\partial}{\partial x_{k}}\left(\overline{u_{i}^{\prime} u_{j}^{\prime}}\right)\right]-C_{p} \frac{\sqrt{\overline{q^{2}}}}{L}\left(\overline{u_{i}^{\prime} u_{j}^{\prime}}-\frac{1}{3} \delta_{i, j} \overline{q^{2}}\right)-C_{v} \frac{1}{L} \delta_{i, j}\left(\overline{q^{2}}\right)^{3 / 2}
\end{aligned}
$$

where $\overline{q^{2}}=2 K=\overline{u_{i}^{\prime} u_{j}^{\prime}}$, and the constants have the following values: $C_{t} \approx 0.30, C_{p} \approx 1.0$ and $C_{v} \approx 1 / 12$. By analogy to equation (6), a density-velocity correlations tensor $\overline{u_{i}^{\prime} \rho^{\prime}}$ is obtained:

$$
\begin{aligned}
\frac{\partial}{\partial t}\left(\overline{u_{i}^{\prime} \rho^{\prime}}\right)+\bar{u}_{j} \frac{\partial}{\partial x_{j}}\left(\overline{u_{i}^{\prime} \rho^{\prime}}\right)=-\overline{u_{i}^{\prime} u_{j}^{\prime}} \frac{\partial \bar{\rho}}{\partial x_{j}}-\overline{u_{j}^{\prime} \rho^{\prime}} \frac{\partial \bar{u}_{i}}{\partial x_{j}} \\
+g_{i} \frac{\overline{\rho^{\prime 2}}}{\rho_{0}}+C_{t} \frac{\partial}{\partial x_{j}}\left[\bar{q} L \frac{\partial}{\partial x_{j}}\left(\overline{u_{i}^{\prime} \rho^{\prime}}\right)\right]-C_{q} \frac{\bar{q}}{L^{\prime}} \overline{u_{i}^{\prime} \rho^{\prime}}
\end{aligned}
$$

with the quadratic term $\overline{\rho^{2}}$ calculated through (8),

$$
\begin{aligned}
\frac{\partial}{\partial t}\left(\overline{\rho^{\prime 2}}\right) & +\bar{u}_{j} \frac{\partial}{\partial x_{j}}\left(\overline{\rho^{\prime 2}}\right)=-2 \overline{u_{j}^{\prime} \rho^{\prime}} \frac{\partial \bar{\rho}}{\partial x_{j}} \\
& +C_{t} \frac{\partial}{\partial x_{j}}\left[\bar{q} L \frac{\partial}{\partial x_{j}}\left(\overline{\rho^{\prime 2}}\right)\right]-C_{r} \frac{\bar{q}}{L}\left(\overline{\rho^{\prime 2}}\right)
\end{aligned}
$$


An equation for the turbulent length scale (or macroscale of the eddies), $L$, is written:

$$
\begin{aligned}
\frac{\partial L}{\partial t} & +\bar{u}_{i} \frac{\partial L}{\partial x_{i}}=C_{l} \frac{L}{\overline{q^{2}}} \overline{u_{i}^{\prime} u_{j}^{\prime}} \frac{\partial \bar{u}_{i}}{\partial x_{j}}+C_{s} \bar{q} \\
& +C_{t} \frac{\partial}{\partial x_{i}}\left[\bar{q} L \frac{\partial L}{\partial x_{i}}\right]-C_{q} \frac{1}{2 \bar{q}}\left(\frac{\partial \bar{q} L}{\partial x_{i}}\right)^{2}+C_{z} \frac{L}{\overline{q^{2}}} g_{i} \frac{\overline{u_{i}^{\prime} \rho}}{\rho_{0}}
\end{aligned}
$$

where $C_{q} \approx 0.75, C_{r} \approx 0.1125, C_{l} \approx 0.35, C_{s} \approx 0.075$ and $C_{z} \approx 0.80$. As can be easily seen, an equation for the turbulent length scale $L$ is, like all other approximations, of the form:

$$
\frac{\partial L}{\partial t}+\bar{u}_{i} \frac{\partial L}{\partial x_{i}}=\text { PRODUCTION + DISSIPATION + DIFFUSION + BUOYANCY }
$$

Modelling the production and dissipation terms by $C_{l} \frac{L}{q^{2}} \overline{u_{i}^{1} u_{j}^{\prime}} \frac{\partial u_{i}}{\partial x_{j}}$ and $C_{s} \bar{q}$, respectively, the diffusion terms by $C_{t} \frac{\partial}{\partial x_{i}}\left[\bar{q} L \frac{\partial L}{\partial x_{i}}\right]-C_{q} \frac{1}{2 \bar{q}}\left(\frac{\partial \bar{q} L}{\partial x_{i}}\right)^{2}$, as suggested by Lewellen (1977), and adding the buoyancy term, the approximation (9) above is newly obtained.

In summary, equations (2), along with equations (6), (7), (8) and (9) for turbulence closure, constitute a complete 3D turbulent boundary layer model with sediment in suspension.

\subsection{Simplified turbulent boundary layer models}

Proceeding with a non-dimensional analysis of the mean flow equations, without sediment in suspension, and considering:

1. A sinusoidal wave $\left(\hat{U}_{w}, T, L_{w}\right)$.

2. The following boundary layer approximations:

- Small boundary layer thickness $\left(z_{\delta}<<\lambda / 2 \pi\right), \lambda$ being the wave length;

- Nikuradse equivalent bottom rugosity much inferior to the boundary layer thickness $\left(k_{N}<<z_{\delta}\right)$.

3. Small wave amplitude and Stokes hypothesis, which assumes that:

- The maximum wave velocity amplitude is much inferior to the celerity $\left(\hat{U}_{w}<<\sqrt{g h}\right)$.

4. Local equilibrium turbulence, along with the turbulent kinetic energy is equivalent to the viscous dissipation. Assuming local equilibrium there is no time evolution or spatial diffusion of the correlations, and the Reynolds stress equation $\overline{u_{i}^{\prime} u_{j}^{\prime}}$ can be reduced.

In summary, assuming these hypotheses we can: $i)$ consider a horizontal flow $(u, v, w=0)$; ii) neglect the convective and horizontal diffusion transport, and iii) simplify the turbulent transport equations, cancelling the remaining time variation terms and the diffusion terms of the velocity correlations.

Considering the above hypotheses in the pure hydrodynamic Reynolds equations (2-b), without stratification, the following approximations (11) result:

$$
\frac{\partial u}{\partial t}=-\frac{1}{\rho_{0}} \frac{\partial P}{\partial x}-\frac{\partial}{\partial z}\left(\overline{u^{\prime} w^{\prime}}\right) ; \frac{\partial v}{\partial t}=-\frac{1}{\rho_{0}} \frac{\partial P}{\partial y}-\frac{\partial}{\partial z}\left(\overline{v^{\prime} w^{\prime}}\right)
$$


On the other hand, under the same assumptions, the Reynolds stress equation $\overline{u_{i}^{\prime} u_{j}^{\prime}}$ (6) can be written explicitly:

$$
\begin{aligned}
& \frac{\partial \overline{u^{\prime} w^{\prime}}}{\partial t}=-\overline{w^{\prime 2}} \frac{\partial u}{\partial z}+C_{t} \frac{\partial}{\partial z}\left(\bar{q} L \frac{\partial \overline{u^{\prime} w^{\prime}}}{\partial z}\right)-C_{p} \frac{q}{L} \overline{u^{\prime} w^{\prime}} \\
& \frac{\partial \overline{v^{\prime} w^{\prime}}}{\partial t}=-\overline{w^{\prime 2}} \frac{\partial v}{\partial z}+C_{t} \frac{\partial}{\partial z}\left(\bar{q} L \frac{\partial \overline{v^{\prime} w^{\prime}}}{\partial z}\right)-C_{p} \frac{q}{L} \overline{v^{\prime} w^{\prime}} \\
& \frac{\partial \overline{u^{\prime 2}}}{\partial t}=-2 \overline{u^{\prime} w^{\prime}} \frac{\partial u}{\partial z}+C_{t} \frac{\partial}{\partial z}\left(\bar{q} L \frac{\partial \overline{u^{\prime 2}}}{\partial z}\right)-C_{p} \frac{q}{L}\left(\overline{u^{\prime 2}}-\frac{q^{2}}{3}\right)-C_{v} \frac{q^{3}}{L} \\
& \frac{\partial \overline{v^{\prime 2}}}{\partial t}=-2 \overline{v^{\prime} w^{\prime}} \frac{\partial v}{\partial z}+C_{t} \frac{\partial}{\partial z}\left(\bar{q} L \frac{\partial \overline{v^{\prime 2}}}{\partial z}\right)-C_{p} \frac{q}{L}\left(\overline{v^{\prime 2}}-\frac{q^{2}}{3}\right)-C_{v} \frac{q^{3}}{L} \\
& \frac{\partial \overline{w^{\prime 2}}}{\partial t}=\quad+C_{t} \frac{\partial}{\partial z}\left(\bar{q} L \frac{\partial \overline{w^{\prime 2}}}{\partial z}\right)-C_{p} \frac{q}{L}\left(\overline{w^{\prime 2}}-\frac{q^{2}}{3}\right)-C_{v} \frac{q^{3}}{L}
\end{aligned}
$$

Adding the last three equations we get (13) for $q^{2}$ :

$$
\frac{\partial q^{2}}{\partial t}=-2 \overline{u^{\prime} w^{\prime}} \frac{\partial u}{\partial z}-2 \overline{v^{\prime} w^{\prime}} \frac{\partial v}{\partial z}+C_{t} \frac{\partial}{\partial z}\left(\bar{q} L \frac{\partial q^{2}}{\partial z}\right)-3 C_{v} \frac{q^{3}}{L}
$$

Taking now into account local equilibrium turbulence (Sheng, 1984), which can be assumed when the scale $L / q$ is much smaller than the time scale of the mean flow and when the turbulent quantities have a small variation on the macroscale of the eddies $L$. In addition, neglecting both variations in time and diffusive transport terms, from equations system (12) the following equations (14) are obtained:

$$
\begin{gathered}
\overline{w^{\prime 2}} \frac{\partial u}{\partial z}+\frac{q}{L} \overline{u^{\prime} w^{\prime}}=0 ; \overline{w^{\prime 2}} \frac{\partial v}{\partial z}+\frac{q}{L} \overline{v^{\prime} w^{\prime}}=0 \\
2 \overline{u^{\prime} w^{\prime}} \frac{\partial u}{\partial z}+\frac{q}{L}\left(\overline{u^{\prime 2}}-\frac{q^{2}}{3}\right)+C_{v} \frac{q^{3}}{L}=0 \\
2 \overline{v^{\prime} w^{\prime}} \frac{\partial v}{\partial z}+\frac{q}{L}\left(\overline{v^{\prime 2}}-\frac{q^{2}}{3}\right)+C_{v} \frac{q^{3}}{L}=0 \\
\frac{q}{L}\left(\overline{w^{\prime 2}}-\frac{q^{2}}{3}\right)+C_{v} \frac{q^{3}}{L}=0
\end{gathered}
$$

This system of equations allows us to obtain (15):

$$
\begin{aligned}
& \overline{u^{\prime 2}}=6 C_{v} L^{2}\left(\frac{\partial u}{\partial z}\right)^{2}+3 C_{v} q^{2} ; \overline{v^{\prime 2}}=6 C_{v} L^{2}\left(\frac{\partial v}{\partial z}\right)^{2}+3 C_{v} q^{2} ; \\
& \overline{w^{\prime 2}}=3 C_{v} q^{2} ;-\overline{u^{\prime} w^{\prime}}=3 C_{v} q L \frac{\partial u}{\partial z} ;-\overline{v^{\prime} w^{\prime}}=3 C_{v} q L \frac{\partial v}{\partial z}
\end{aligned}
$$


Comparing the last two equations of (15) with $-\overline{u^{\prime} w^{\prime}}=v_{t} \frac{\partial u}{\partial z}$ and $-\overline{v^{\prime} w^{\prime}}=v_{t} \frac{\partial v}{\partial z}$ it is clear that:

$$
v_{\mathrm{t}}=3 C_{v} q L=3 C_{v} \sqrt{2 K} L=\frac{\sqrt{2 K} L}{4}
$$

In addition, it can be seen from the first three equations of (15) that:

$$
q^{2}=2 K=24 C_{v} L^{2}\left[\left(\frac{\partial u}{\partial z}\right)^{2}+\left(\frac{\partial v}{\partial z}\right)^{2}\right]=2 L^{2}\left[\left(\frac{\partial u}{\partial z}\right)^{2}+\left(\frac{\partial v}{\partial z}\right)^{2}\right]
$$

\subsection{DV turbulent boundary layer models}

Considering now a horizontal flow along $x$-direction $(u, v=0, w=0)$ with sediment in suspension, so with the buoyancy terms, and local equilibrium turbulence, the system (12) is written in the following form (18):

$$
\begin{array}{lll}
\frac{\partial \overline{u^{\prime} w^{\prime}}}{\partial t}=0=-\overline{w^{\prime 2}} \frac{\partial u}{\partial z} & -\frac{g}{\rho_{0}} \overline{u^{\prime} \rho^{\prime}}-C_{p} \frac{q}{L} \overline{u^{\prime} w^{\prime}} & \\
\frac{\partial \overline{w^{\prime 2}}}{\partial t}=0= & -\frac{2 g}{\rho_{0}} \overline{w^{\prime} \rho^{\prime}}-C_{p} \frac{q}{L}\left(\overline{w^{\prime 2}}-\frac{q^{2}}{3}\right)-C_{v} \frac{q^{3}}{L} \\
\frac{\partial \overline{u^{\prime} \rho^{\prime}}}{\partial t}=0=-\overline{w^{\prime} \rho^{\prime}} \frac{\partial u}{\partial z}-\overline{u^{\prime} w^{\prime}} \frac{\partial \rho}{\partial z} & -C_{q} \frac{q}{L} \overline{u^{\prime} \rho^{\prime}} \\
\frac{\partial \overline{w^{\prime} \rho^{\prime}}}{\partial t}=0=-\overline{w^{\prime 2}} \frac{\partial \rho}{\partial z} & -\frac{g}{\rho_{0}} \overline{\rho^{\prime 2}} & -C_{q} \frac{q}{L} \overline{w^{\prime} \rho^{\prime}} \\
\frac{\partial \overline{\rho^{\prime 2}}}{\partial t}=0=-2 \overline{w^{\prime} \rho^{\prime}} \frac{\partial \rho}{\partial z} & -C_{r} \frac{q}{L} \overline{\rho^{\prime 2}}
\end{array}
$$

Solving this equation system for $\overline{u^{\prime} w^{\prime}}$ and $\overline{w^{\prime} \rho^{\prime}}$ we obtain (19):

$$
\overline{u^{\prime} w^{\prime}}=-\frac{(1-16.444 \Omega)}{(1-19.778 \Omega)(1-\Omega)} \frac{q L}{4} \frac{\partial u}{\partial z} ; \overline{w^{\prime} \rho^{\prime}}=-\frac{1}{(1-19.778 \Omega)} \frac{q L}{3} \frac{\partial \rho}{\partial \mathbf{z}}
$$

where $\Omega=\frac{4}{3} \frac{g}{\rho_{0}} \frac{\partial \rho}{\partial z} \frac{L^{2}}{q^{2}}$. As shown before and from (3) $-\overline{u^{\prime} w^{\prime}}=v_{t} \frac{\partial u}{\partial z}$ and $\overline{-w^{\prime} \rho^{\prime}}=\gamma_{t} \frac{\partial \bar{\rho}}{\partial z}$; comparing with the expressions (19) above we can write (20) and (21):

$$
\begin{gathered}
v_{t}=\frac{(1-16.444 \Omega)}{(1-19.778 \Omega)(1-\Omega)} \frac{q L}{4}=\frac{(1-16.444 \Omega)}{(1-19.778 \Omega)(1-\Omega)} \frac{\sqrt{2 K} L}{4} \\
\gamma_{t}=\frac{1}{(1-19.778 \Omega)} \frac{q L}{3}=\frac{(1-\Omega)}{(1-16.444 \Omega)} \frac{4}{3} v_{t}
\end{gathered}
$$




\subsubsection{Two-equation K-L 1DV boundary layer model}

Taking into account the assumptions stated before, a complete set of governing equations (22) for the two-equation $K-L$ model is written (Tran-Thu \& Temperville, 1994):

$$
\begin{gathered}
\frac{\partial u}{\partial t}=-\frac{1}{\rho_{0}} \frac{\partial P}{\partial x}+\frac{\partial}{\partial z}\left(v_{t} \frac{\partial u}{\partial z}\right) ; \frac{\partial v}{\partial t}=-\frac{1}{\rho_{0}} \frac{\partial P}{\partial y}+\frac{\partial}{\partial z}\left(v_{t} \frac{\partial v}{\partial z}\right) \\
\underbrace{\frac{\partial K}{\partial t}}_{\begin{array}{c}
\text { rate of } \\
\text { change }
\end{array}}=\underbrace{v_{t}\left[\left(\frac{\partial u}{\partial z}\right)^{2}+\left(\frac{\partial v}{\partial z}\right)^{2}\right]}_{\text {production }}-\underbrace{\frac{\sqrt{2 K}}{4 L} K}_{\text {dissipation }}+\underbrace{0.30 \frac{\partial}{\partial z}\left(\sqrt{2 K} L \frac{\partial K}{\partial z}\right)}_{\text {diffusion }}+\underbrace{\frac{g}{\rho_{0}} \gamma_{t} \frac{\partial \rho}{\partial z}}_{\text {buoyancy }} \\
\underbrace{\frac{\partial L}{\partial t}}_{\begin{array}{l}
\text { rate of } \\
\text { change }
\end{array}}=\underbrace{-0.35 \frac{v_{t}}{2 K}\left[\left(\frac{\partial u}{\partial z}\right)^{2}+\left(\frac{\partial v}{\partial z}\right)^{2}\right] L}_{\text {production }}+\underbrace{0.075 \sqrt{2 K}}_{\text {dissipation }} \\
+\underbrace{0.30 \frac{\partial}{\partial z}\left(\sqrt{2 K} L \frac{\partial L}{\partial z}\right)-\frac{0.375}{\sqrt{2 K}}\left[\frac{\partial}{\partial z}(\sqrt{2 K} L)\right]^{2}}_{\frac{\partial C}{\partial t}=\frac{\partial\left(w_{s} C\right)}{\partial z}+\frac{\partial}{\partial z}\left(\gamma_{t} \frac{\partial C}{\partial z}\right)}+\underbrace{0.80 \frac{L}{2 K} \frac{g}{\rho_{0}} \gamma_{t} \frac{\partial \rho}{\partial z}}_{\text {buoyancy }}
\end{gathered}
$$

where $u$ and $v$ are horizontal components of flow velocity in the boundary layer; $C$ is the volumetric concentration; $w_{s}$ is the sediment settling velocity; $K$ is the turbulent kinetic energy, and $L$ is the length scale of the large vortices.

The turbulent viscosity $v_{t}$ and the turbulent diffusivity $\gamma_{t}$ are given by equations (20) and (21), respectively. The hydrodynamic equations and the concentration equation are coupled through the equation (23) for the density:

$$
\rho=\rho_{0}+\left(\rho_{s}-\rho_{0}\right) C
$$

where $\rho_{0}$ and $\rho_{s}$ are the densities of the fluid and sediment, respectively.

\subsubsection{One-equation K-L 1DV boundary layer model}

With $L=f(k, z, K)$, a complete one-equation $K-L$ turbulence closure model is simply written:

$$
\begin{aligned}
& \frac{\partial u}{\partial t}=-\frac{1}{\rho_{0}} \frac{\partial P}{\partial x}+\frac{\partial}{\partial z}\left(v_{t} \frac{\partial u}{\partial z}\right) ; \frac{\partial v}{\partial t}=-\frac{1}{\rho_{0}} \frac{\partial P}{\partial y}+\frac{\partial}{\partial z}\left(v_{t} \frac{\partial v}{\partial z}\right) \\
& \frac{\partial K}{\partial t}=v_{t}\left[\left(\frac{\partial u}{\partial z}\right)^{2}+\left(\frac{\partial v}{\partial z}\right)^{2}\right]-\frac{\sqrt{2 K}}{4 L} K+0.30 \frac{\partial}{\partial z}\left(\sqrt{2 K} L \frac{\partial K}{\partial z}\right)+\frac{g}{\rho_{0}} \gamma_{t} \frac{\partial \rho}{\partial z} \\
& L=f(k, z, K) ; \quad \frac{\partial C}{\partial t}=\frac{\partial\left(w_{s} C\right)}{\partial z}+\frac{\partial}{\partial z}\left(\gamma_{t} \frac{\partial C}{\partial z}\right)
\end{aligned}
$$

where (20), (21) and (23) apply. 
A number of empirical equations for the length scale $L$ could be found in the literature; some examples are (with $k=0.4$ ):

- $\quad L=k \sqrt[4]{c_{l} z}$, where $c_{l}=0.08$.

- $\quad L=k z \sqrt{1-z / z_{\delta}}, z_{\delta}$ being the boundary layer thickness.

- $L=k \sqrt{K}\left\{\int_{z_{o}}^{z} K^{-1 / 2} d z+z_{o} K_{o}^{-1 / 2}\right\}, K_{o}=K\left(z_{o}, t\right)$.

- $L=k z_{w}\left(1-e^{z_{w}^{+}} / A\right)$, where $A \approx 26, z_{w}$ is the distance to the wall and $z_{w}^{+}=\left(z_{w} u_{T}\right) / v_{l}$, $u_{T}$ being the friction velocity.

The influence of a stable stratification on $L$ can be taken into account through (25),

$$
\left(\frac{L}{L_{o}}\right)^{2}=\left(1+\beta R_{i}\right)^{n}, \text { with } \beta \approx(10,14) \text { and } n \approx(-0.5,-1.5)
$$

where $R_{i}=-\frac{g}{\rho} \frac{\partial \rho}{\partial z} /\left[\left(\frac{\partial u}{\partial z}\right)^{2}+\left(\frac{\partial v}{\partial z}\right)^{2}\right]$ is the Richardson number and $L_{o}$ is the length scale $L$ value without stratification.

\subsubsection{Zero-equation boundary layer model}

Defining the mixing length as $l_{m}=k z \sqrt{1-z / z_{\delta}}$, where $k \approx 0.4$ and $z_{\delta}$ is the boundary layer thickness, equations (26) for the $u$ and $v$ variables in the boundary layer are obtained:

$$
\frac{\partial u}{\partial t}=-\frac{1}{\rho_{0}} \frac{\partial P}{\partial x}+\frac{\partial}{\partial z}\left(l_{m}^{2}\left|\frac{\partial u}{\partial z}\right| \frac{\partial u}{\partial z}\right) ; \frac{\partial v}{\partial t}=-\frac{1}{\rho_{0}} \frac{\partial P}{\partial y}+\frac{\partial}{\partial z}\left(l_{m}^{2}\left|\frac{\partial v}{\partial z}\right| \frac{\partial v}{\partial z}\right)
$$

Stable stratification effects on $l_{m}$ could be taken into account through the relation $\left(l_{m} / l_{m o}\right)^{2}=\left(1+10 R_{i}\right)^{-0.5}$, where $l_{m 0}$ is the mixing length $l_{m}$ value without stratification, and $R_{i}$ is the Richardson number, as defined above. We now assume in (26):

$$
-\frac{1}{\rho_{0}} \frac{\partial P}{\partial x}=\frac{\partial U}{\partial t} \text { and }-\frac{1}{\rho_{0}} \frac{\partial P}{\partial y}=\frac{\partial V}{\partial t}
$$

where $U$ and $V$ are the velocity components outside of the boundary layer. Defining the deficit velocity components $\left(u_{d}, v_{d}\right)$ as (28),

$$
u_{d}(z, t)=u(z, t)-U(t) ; v_{d}(z, t)=v(z, t)-V(t)
$$

and substituting in (26) the following equations (29) are obtained,

$$
\frac{\partial u_{d}}{\partial t}=\frac{\partial}{\partial z}\left(l_{m}^{2}\left|\frac{\partial u_{d}}{\partial z}\right| \frac{\partial u_{d}}{\partial z}\right) ; \frac{\partial v_{d}}{\partial t}=\frac{\partial}{\partial z}\left(l_{m}^{2}\left|\frac{\partial v_{d}}{\partial z}\right| \frac{\partial v_{d}}{\partial z}\right)
$$

These equations are non-linear and no analytical solutions are available, so they have to be solved numerically, as will be shown later. 


\subsection{Boundary conditions for 1DV turbulent boundary layer models}

\subsubsection{One- and two-equation boundary layer models of the K-L type}

- At the lower limit of the boundary layer, $z=z_{0}$

- $u\left(z_{0}\right)=v\left(z_{0}\right)=0 ; \partial K / \partial z=0 ; L\left(z_{0}\right)=\alpha z_{0}$, with $\alpha=0.67$ (empirical constant).

- At the hydraulic rough regime, the level $z_{0}$ is taken to be $k_{N} / 30$, with $k_{N}=2.5 d$ the Nikuradse equivalent roughness of a bed of sand with diameter $d$. In the transitory regime, $k_{N}$ and $z_{0}$ are calculated following Sleath (1984) (Tran Thu and Temperville, 1994).

- For the reference concentration at the bottom, $C_{b}$, the following relations may be used: $C=C_{b} \approx 0.63$, or $C=C(\psi)$, where $\psi=\tau_{b}(t) /(\rho(s-1) g d)$.

- At the upper limit of the boundary layer, $z=z_{\delta}$

Assuming that the instantaneous velocity $\vec{U}(t)$ is given at a level $z=z_{\delta}$ outside the boundary layer, the boundary conditions are:

- $\quad \vec{u}\left(z_{\delta}\right)=\vec{U}(t), \vec{U}(t)$ may contain a component of the mean current $U_{c}$ as well as oscillatory components of the wave;

- $K\left(z_{\delta}\right)=0$ (pure wave), or $\partial K /\left.\partial z\right|_{z_{\delta}}=0$ (combined wave and current);

- $\quad L\left(z_{\delta}\right)=0$ (pure wave), or $L\left(z_{\delta}\right)=\alpha z_{\delta}$ (combined wave and current);

- Depending on the problem, the condition $\partial L /\left.\partial z\right|_{z_{\delta}}=0$ may be also adequate;

- $C\left(z_{\delta}\right)=0$ (pure wave), or $w_{q} C+\gamma_{t} \partial C /\left.\partial t\right|_{z}=0$ (combined wave and current).

Initial values for $u, v, K$ and $L$ are the solution for the initial field current velocities $\left(U_{c}\right.$, $\left.V_{c}\right)$.

- Estimation of the boundary layer thickness, $z_{\delta}$

Considering a pure current $\left(\hat{U}_{w}=0\right)$ in a channel with a water column $h$, the boundary layer thickness is $z_{\delta}=h$.

Assuming now a pure wave $\left(U_{c}=V_{c}=0\right)$ propagating in a channel, the boundary layer thickness reaches its minimum value and can be approximated by $z_{\delta} / k_{N}=0.246\left(\hat{a} / k_{N}\right)^{0.81}$ (Huynh-Thanh, 1990), where the orbital amplitude is given by $\hat{a}=\hat{U}_{\text {weq }} T_{c h} /(2 \pi)$ for an equivalent sinusoidal wave with $\hat{U}_{\text {weq }}$, and during a characteristic signal period $T_{c h}$ (Antunes do Carmo et al., 1996). The relation proposed for $z_{\delta} / k_{N}$ corresponds to the thickness beyond which $K$ is zero.

A general rough estimation for $z_{\delta}$ can be obtained by (30):

$$
z_{\delta}=\frac{\left|\vec{U}_{c}\right| h+\left|\hat{U}_{w}\right| z_{\delta w}}{\left|\vec{U}_{c}\right|+\left|\hat{U}_{w}\right|}=>z_{\delta}=\frac{\left|\vec{U}_{c}\right| h+0.246 k_{N}\left(\hat{a} / k_{N}\right)^{0.81}\left|\hat{U}_{w}\right|}{\left|\vec{U}_{c}\right|+\left|\hat{U}_{w}\right|}
$$

\subsubsection{Zero-equation model}

The following conditions (31) are imposed at the lower limit $z=z_{0}$ and at the upper limit $z=z_{\delta}$ of the boundary layer:

$$
u_{d}\left(z_{o}, t\right)=-U ; u_{d}\left(z_{\delta}, t\right)=0 ; v_{d}\left(z_{o}, t\right)=-V ; v_{d}\left(z_{\delta}, t\right)=0
$$




\subsection{DV turbulent boundary layer model}

Over movable beds, the interaction of flow and sediment transport creates a variety of bed forms such as ripples, dunes, antidunes or other irregular shapes and obstacles. Their presence, in general, causes flow separation and recirculation, which can alter the overall flow resistance and, consequently, can affect sediment transport within the water mass and bottom erosion. For dunes, in particular, the flow is characterized by an attached flow on their windward side, separation at their crest and formation of a recirculation eddy in their leeside (Fourniotis et al., 2006). A detailed description of the flow over a dune is then of fundamental interest because the pressure and friction (shear-stress) distributions on the bed determine the total resistance on the bottom and the rate of sediment transport. Over bed forms a $1 \mathrm{DV}$ version of the turbulent boundary layer is no able to describe the main processes that occur above and close to the bed surface. Consequently, a 2DV turbulent boundary layer model is developed herein.

Considering a two-dimensional mean non-stratified flow in the vertical plane $(u, v=0, w)$, only non-zero $y$-derivatives are present. The physical problem is outlined in figure 1 below, under the action of a wave. Knowing that the wavelength is always greater than the length of the ripples, i.e. $L_{w}>>L_{r}$, we can restrict the domain of calculation, instead of investigate all the domain over of the whole wavelength.

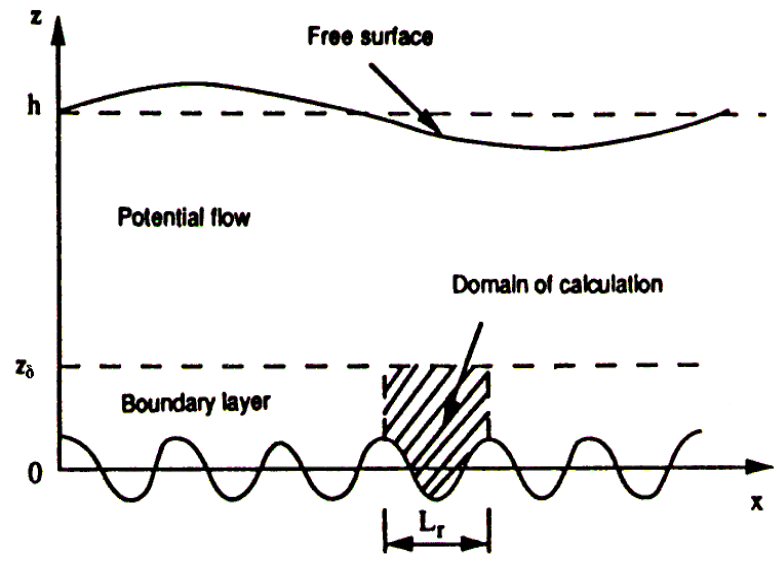

Fig. 1. Scheme of the physical system (Huynh-Thanh \& Temperville, 1991)

The basic equations of the model are derived from the previous ones (2). In order to simplify the numerical resolution of the equations we make use of the stream function $(\Psi)$ and vorticity $(\xi)$ variables, instead of the velocities $u$ and $v$, and a transformation of the physical domain into a rectangular one. Considering that only two-independent spatial derivatives are involved in the flow, in the $x z$-plane, i.e., a flow with only velocity components $u(x, z, t)$ and $w(x, z, t)$, the equations of motion are restricted to the continuity equation and the two components of the Reynolds equations. Under these assumptions, from (2) the two components (32) and (33) of the pure hydrodynamic momentum equation are written:

$$
\frac{\partial u}{\partial t}+u \frac{\partial u}{\partial x}+w \frac{\partial u}{\partial z}=-\frac{1}{\rho} \frac{\partial p}{\partial x}+\frac{\partial}{\partial x}\left(-\overline{u^{\prime 2}}\right)+\frac{\partial}{\partial z}\left(-\overline{u^{\prime} w^{\prime}}\right)
$$




$$
\frac{\partial w}{\partial t}+u \frac{\partial w}{\partial x}+w \frac{\partial w}{\partial z}=-\frac{1}{\rho} \frac{\partial p}{\partial z}+\frac{\partial}{\partial x}\left(-\overline{u^{\prime} w^{\prime}}\right)+\frac{\partial}{\partial z}\left(-\overline{w^{\prime 2}}\right)
$$

Substituting in (32) and (33) the approximations (34),

$$
-\overline{u^{\prime 2}}=2 v_{t} \frac{\partial u}{\partial x} ; \overline{-\overline{u^{\prime} w^{\prime}}}=v_{t}\left(\frac{\partial u}{\partial z}+\frac{\partial w}{\partial x}\right) \text { and }-\overline{w^{\prime 2}}=2 v_{t} \frac{\partial w}{\partial z}
$$

the governing equations (35) and (36) result:

$$
\begin{aligned}
& \frac{\partial u}{\partial t}+u \frac{\partial u}{\partial x}+w \frac{\partial u}{\partial z}=-\frac{1}{\rho} \frac{\partial p}{\partial x}+2 \frac{\partial}{\partial x}\left(v_{t} \frac{\partial u}{\partial x}\right)+\frac{\partial}{\partial z}\left[v_{t}\left(\frac{\partial u}{\partial z}+\frac{\partial w}{\partial x}\right)\right] \\
& \frac{\partial w}{\partial t}+u \frac{\partial w}{\partial x}+w \frac{\partial w}{\partial z}=-\frac{1}{\rho} \frac{\partial p}{\partial z}+\frac{\partial}{\partial x}\left[v_{t}\left(\frac{\partial u}{\partial z}+\frac{\partial w}{\partial x}\right)\right]+2 \frac{\partial}{\partial z}\left(v_{t} \frac{\partial w}{\partial z}\right)
\end{aligned}
$$

The unknown pressure gradient due to the bed forms can now be eliminated from equations (35) and (36) by cross-differentiation, i.e., taking the curl of the two-dimensional vector momentum equations. The result reads:

$$
\begin{aligned}
& \frac{\partial}{\partial t}\left(\frac{\partial u}{\partial z}-\frac{\partial w}{\partial x}\right)+u \frac{\partial}{\partial x}\left(\frac{\partial u}{\partial z}-\frac{\partial w}{\partial x}\right)+w \frac{\partial}{\partial z}\left(\frac{\partial u}{\partial z}-\frac{\partial w}{\partial x}\right)= \\
& \quad-\frac{\partial^{2}}{\partial x^{2}}\left[v_{t}\left(\frac{\partial u}{\partial z}+\frac{\partial w}{\partial x}\right)\right]+2 \frac{\partial^{2}}{\partial x \partial z}\left[v_{t}\left(\frac{\partial u}{\partial x}-\frac{\partial w}{\partial z}\right)\right]+\frac{\partial^{2}}{\partial z^{2}}\left[v_{t}\left(\frac{\partial u}{\partial z}+\frac{\partial w}{\partial x}\right)\right]
\end{aligned}
$$

By definition, the following relations (38) account:

$$
u=\frac{\partial \Psi}{\partial z} ; w=-\frac{\partial \Psi}{\partial x} ; \xi=\frac{\partial u}{\partial z}-\frac{\partial w}{\partial x}
$$

Inserting the stream function $(\Psi)$ and vorticity $(\xi)$ variables in equation (37) the following result (39) for the vorticity is obtained (Huynh-Thanh, 1990; Tran-Thu, 1995):

$$
\frac{\partial \xi}{\partial t}-\frac{\partial(\Psi, \xi)}{\partial(x, z)}=\nabla^{2}\left(v_{t} \xi\right)-2\left(\frac{\partial^{2} v_{t}}{\partial x^{2}} \frac{\partial^{2} \Psi}{\partial z^{2}}-2 \frac{\partial^{2} v_{t}}{\partial x \partial z} \frac{\partial^{2} \Psi}{\partial x \partial z}+\frac{\partial^{2} v_{t}}{\partial z^{2}} \frac{\partial^{2} \Psi}{\partial x^{2}}\right)
$$

where $\frac{\partial(\Psi, \xi)}{\partial(x, z)}=\frac{\partial \Psi}{\partial x} \frac{\partial \xi}{\partial z}-\frac{\partial \Psi}{\partial z} \frac{\partial \xi}{\partial x}$ and $\nabla^{2}=\frac{\partial^{2}}{\partial x^{2}}+\frac{\partial^{2}}{\partial z^{2}}$.

An equation for the stream function is obtained through the definitions (38), substituting $u$ and $v$ in $\xi$ :

$$
\nabla^{2} \Psi=\xi
$$

which is known as the Poisson equation. The turbulent viscosity $v_{t}$ is obtained assuming local equilibrium turbulence. Once more in the vertical plane $(u, v=0, w)$, the following equations system (41) can be written: 


$$
\begin{array}{ll}
\frac{\partial \overline{u^{\prime} w^{\prime}}}{\partial t}=0=-\left(\overline{u^{\prime 2}} \frac{\partial w}{\partial x}+\overline{w^{\prime 2}} \frac{\partial u}{\partial z}\right) & -C_{p} \frac{q}{L} \overline{u^{\prime} w^{\prime}} \\
\frac{\partial \overline{u^{\prime 2}}}{\partial t}=0=-2\left(\overline{u^{\prime 2}} \frac{\partial u}{\partial x}+\overline{u^{\prime} w^{\prime}} \frac{\partial u}{\partial z}\right) & -C_{p} \frac{q}{L}\left(\overline{u^{\prime 2}}-\frac{q^{2}}{3}\right)-C_{v} \frac{q^{3}}{L} \\
\frac{\partial \overline{v^{\prime 2}}}{\partial t}=0= & -C_{p} \frac{q}{L}\left(\overline{v^{\prime 2}}-\frac{q^{2}}{3}\right)-C_{v} \frac{q^{3}}{L} \\
\frac{\partial \overline{w^{\prime 2}}}{\partial t}=0=-2\left(\overline{u^{\prime} w^{\prime}} \frac{\partial w}{\partial x}+\overline{w^{\prime 2}} \frac{\partial w}{\partial z}\right)-C_{p} \frac{q}{L}\left(\overline{w^{\prime 2}}-\frac{q^{2}}{3}\right)-C_{v} \frac{q^{3}}{L}
\end{array}
$$

The third equation of this system allows us to obtain $\overline{v^{\prime 2}}=q^{2} / 4=K / 2$, with $C_{p}=1.0$ and $C_{v}=1 / 12$.

Assuming identical production along both $x$ - and $z$-directions, from the second and fourth equations we find that $\overline{u^{\prime 2}}=\overline{w^{\prime 2}}$. This hypothesis is supported by laboratory experiments over a bottom with ripples conducted by Sato et al. (1984), among others. Therefore, as $q^{2}=2 K=\overline{u^{\prime 2}}+\overline{v^{\prime 2}}+\overline{w^{\prime 2}}$, the above results show that $\overline{u^{\prime 2}}=\overline{w^{\prime 2}}=3 q^{2} / 8=3 K / 4$. On the other hand, from the first equation of the system (41) we find that:

$$
-\overline{u^{\prime} w^{\prime}}=\frac{L}{q}\left(\overline{u^{\prime 2}} \frac{\partial w}{\partial x}+\overline{w^{\prime 2}} \frac{\partial u}{\partial z}\right)=\frac{L}{q} \frac{3 q^{2}}{8}\left(\frac{\partial w}{\partial x}+\frac{\partial u}{\partial z}\right)=\frac{3}{8} \sqrt{2 K} L\left(\frac{\partial w}{\partial x}+\frac{\partial u}{\partial z}\right)
$$

Therefore,

$$
v_{t}=\frac{3}{8} \sqrt{2 K} L
$$

The equation for the turbulent kinetic energy, $K$, is obtained through the earlier already presented in two-dimensions in the vertical plane:

$$
\begin{aligned}
\frac{\partial K}{\partial t}+u \frac{\partial K}{\partial x} & +w \frac{\partial K}{\partial z}=v_{t}\left[2\left(\frac{\partial u}{\partial x}\right)^{2}+\left(\frac{\partial u}{\partial z}+\frac{\partial w}{\partial x}\right)^{2}+2\left(\frac{\partial w}{\partial z}\right)^{2}\right] \\
& -\frac{K \sqrt{2 K}}{4 L}+0.30 \frac{\partial}{\partial x}\left(\sqrt{2 K} L \frac{\partial K}{\partial x}\right)+0.30 \frac{\partial}{\partial z}\left(\sqrt{2 K} L \frac{\partial K}{\partial z}\right)
\end{aligned}
$$

Inserting the stream function ( $\Psi$ ) in equation (44), we find (45):

$$
\begin{aligned}
\frac{\partial K}{\partial t}-\underbrace{\frac{\partial(\Psi, K)}{\partial(x, z)}=}_{\text {advection }} & \underbrace{v_{t}\left[4\left(\frac{\partial^{2} \Psi}{\partial x \partial z}\right)^{2}+\left(\frac{\partial^{2} \Psi}{\partial z^{2}}-\frac{\partial^{2} \Psi}{\partial x^{2}}\right)^{2}\right]}_{\text {production }} \\
& -\underbrace{\frac{2}{3} v_{t} \frac{K}{L^{2}}}_{\text {dissipation }}+\underbrace{0.80 \frac{\partial}{\partial x}\left(v_{t} \frac{\partial K}{\partial x}\right)+0.80 \frac{\partial}{\partial z}\left(v_{t} \frac{\partial K}{\partial z}\right)}_{\text {diffusion }}
\end{aligned}
$$


The length scale $L$ is directly imposed by the analytical solution (46):

$$
L=0.67 z \sqrt{1-z / z_{\delta}}
$$

In order to describe the space-time distribution of the sediments concentration over a bottom with ripples, an equation for $C$ is included, considering in it the advection and diffusion terms in both $\mathrm{x}$-horizontal and z-vertical directions:

$$
\frac{\partial C}{\partial t}+\frac{\partial}{\partial x}(u C)+\frac{\partial}{\partial z}\left[\left(w-w_{s}\right) C\right]=\frac{\partial}{\partial x}\left(\gamma_{t} \frac{\partial C}{\partial x}\right)+\frac{\partial}{\partial z}\left(\gamma_{t} \frac{\partial C}{\partial z}\right)
$$

In order to simplify the numerical resolution of the equations, as well as the description of the boundary conditions at the ripples surface, the physical domain in coordinates $(x, z)$ is transformed into a rectangular one (the computation domain) utilizing orthogonal curvilinear coordinates $(X, Z)$ (Figure 2), using the following transformations (48) (Sato et al., 1984; Huynh-Thanh, 1990; Tran-Thu, 1995; Silva, 2001):

$$
\begin{aligned}
& X=x+\sum_{n=1}^{N} a_{n} \exp \left(-n \frac{2 \pi}{L_{r}} Z\right) \sin \left(n \frac{2 \pi}{L_{r}} X-\theta_{n}\right) \\
& Z=z-\sum_{n=1}^{N} a_{n} \exp \left(-n \frac{2 \pi}{L_{r}} Z\right) \cos \left(n \frac{2 \pi}{L_{r}} X-\theta_{n}\right)
\end{aligned}
$$

where $N, a_{n}$ and $\theta_{n}$ are coefficients to be determined in such a way that the curve $Z=0$ represents the real ripple.
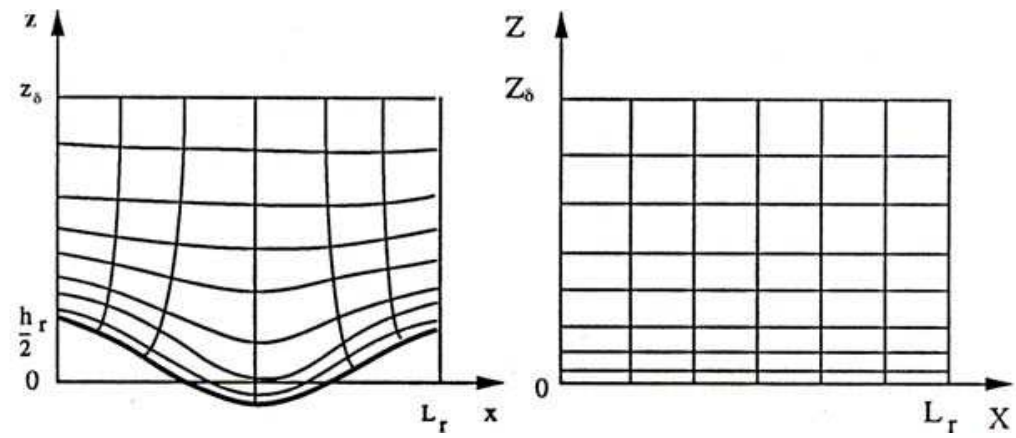

Fig. 2. Physical and computational domains. Transformation of coordinates $(x, z) \rightarrow(X, Z)$

The Jacobian of the transformation is defined by (49):

$$
\boldsymbol{J}=\frac{\partial(X, Z)}{\partial(x, z)}=\frac{\partial X}{\partial x} \frac{\partial Z}{\partial z}-\frac{\partial X}{\partial z} \frac{\partial Z}{\partial x}=\left(\frac{\partial X}{\partial x}\right)^{2}+\left(\frac{\partial X}{\partial z}\right)^{2}
$$

which is calculated from the inverse transformation of the Jacobian $\boldsymbol{J}_{0}\left(\boldsymbol{J}=\boldsymbol{J}_{0}^{-1}\right)$. After carried out the transformation of coordinates $(x, z) \rightarrow(X, Z)$, the above equations (39), (40), (43), (45) and (47) are written and solved iteratively as will be shown later (see HuynhThanh, 1990, Tran-Thu, 1995, and Silva, 2001, for details): 


$$
\begin{gathered}
\frac{\partial \xi}{\partial t}-\boldsymbol{J} \frac{\partial(\Psi, \xi)}{\partial(X, Z)}=\boldsymbol{J} \nabla^{2}\left(v_{t} \xi\right)-2\left(\frac{\partial^{2} v_{t}}{\partial z^{2}} \frac{\partial^{2} \Psi}{\partial x^{2}}-2 \frac{\partial^{2} v_{t}}{\partial x \partial z} \frac{\partial^{2} \Psi}{\partial x \partial z}+\frac{\partial^{2} v_{t}}{\partial x^{2}} \frac{\partial^{2} \Psi}{\partial z^{2}}\right) \\
\boldsymbol{J} \nabla_{\mathrm{XZ}}^{2} \Psi=\xi \\
v_{t}=\frac{3}{8} \sqrt{2 K} L
\end{gathered}
$$

where an algebraic equation for $L$ is used, $L=0.67 Z \sqrt{1-Z / z_{\delta}}$. For $K$ we get (53),

$$
\frac{\partial K}{\partial t}-\boldsymbol{J} \frac{\partial(\Psi, K)}{\partial(X, Z)}=0.80 \boldsymbol{J}\left[\frac{\partial}{\partial X}\left(v_{t} \frac{\partial K}{\partial X}\right)+\frac{\partial}{\partial Z}\left(v_{t} \frac{\partial K}{\partial Z}\right)\right]-\frac{2}{3} v_{t} \frac{K}{L^{2}}+\boldsymbol{P}
$$

where $\boldsymbol{P}=v_{t}\left[4\left(\frac{\partial^{2} \Psi}{\partial x \partial z}\right)^{2}+\left(\frac{\partial^{2} \Psi}{\partial z^{2}}-\frac{\partial^{2} \Psi}{\partial x^{2}}\right)^{2}\right]$ represents the production of $K$, and for C:

$$
\frac{\partial C}{\partial t}+\boldsymbol{J} \frac{\partial}{\partial X}\left[\left(\frac{\partial \psi}{\partial Z}+w_{s} \frac{\partial x}{\partial Z}\right) C-\gamma_{t} \frac{\partial C}{\partial X}\right]-\boldsymbol{J} \frac{\partial}{\partial Z}\left[\left(\frac{\partial \psi}{\partial X}+w_{s} \frac{\partial x}{\partial X}\right) C+\gamma_{t} \frac{\partial C}{\partial Z}\right]=0
$$

\subsection{Boundary conditions for a 2DV turbulent boundary layer model}

- At the lower limit of the boundary layer, $z=z_{0}=k_{N} / 30$

- conditions for the stream current: $\partial \Psi / \partial X=\partial \Psi / \partial Z=0 ; \Psi=0$.

- $\quad$ condition for the turbulent kinetic energy: $\partial K / \partial Z=0$.

- condition for the vorticity: $\xi_{0}=2 J \Psi_{1} /\left(Z_{1}-Z_{0}\right)^{2}$, where $\Psi_{1}$ is the stream function value at height $Z_{1}$. Value for $\xi_{0}$ can be also obtained from the one obtained at the time precedent through $\xi=J \nabla_{X Z}^{2} \Psi=J \partial^{2} \Psi / \partial Z^{2}$.

- At the upper limit of the boundary layer, $z=z_{\delta}$

- condition for the stream current: $\partial \Psi / \partial Z=U(t)$, where $U(t)=U_{c}+U_{w} \sin (\omega t)$, or $\Psi\left(z_{\delta}, t\right)=Q(t)$ if the flow is known at the level $z=z_{\delta}$.

- condition for the turbulent kinetic energy. $K=0$ (pure current), or $\partial K / \partial Z=0$ (combined wave and current).

- condition for the vorticity: $\xi=0$ (it is assumed non-rotational flow outside of the boundary layer).

At the lateral boundaries $(X=0$ and $X=L)$, a spatially periodic condition for $\Psi, \xi$ and $K$ is assumed.

\subsection{Other simplified two-equation turbulence closure models}

A relation for the turbulent viscosity, equivalent to (16), can be written as $v_{t}=C_{\mu} K^{2} / \mathcal{\varepsilon}$, where $\varepsilon$ is the turbulent dissipation rate defined by $\varepsilon=v_{l} \overline{\frac{\partial u_{i}^{\prime}}{\partial x_{k}} \frac{\partial u_{j}^{\prime}}{\partial x_{k}}}$. Comparing this definition of the eddy viscosity $v_{t}$ with (16), a relation between $L$ and $\varepsilon$ is found 
$\varepsilon=C_{K} K^{3 / 2} / L$. Any other combination of the form $K^{m} L^{n}$ can be utilized, for example the specific dissipation rate $\omega=C_{\omega L} K^{1 / 2} / L=\varepsilon /\left(C_{\omega \varepsilon} K\right)$. This suggests the use of different variables, other than the macroscale of the eddies $L$, with all approximations of the form (10). One of these turbulence closure schemes, possibly the best known, is the two-equation $K-\varepsilon$ model; its governing equations are written:

$$
\begin{gathered}
\frac{\partial K}{\partial t}+\bar{u}_{j} \frac{\partial K}{\partial x_{j}}=\frac{\partial}{\partial x_{j}}\left(\frac{v_{t}}{\sigma_{K}} \frac{\partial K}{\partial x_{j}}\right)+v_{t}\left(\frac{\partial \bar{u}_{i}}{\partial \mathrm{x}_{j}}+\frac{\partial \bar{u}_{j}}{\partial x_{i}}\right) \frac{\partial \bar{u}_{i}}{\partial \mathrm{x}_{j}}-\varepsilon ; i, j=1,2,3 \\
\frac{\partial \varepsilon}{\partial t}+\bar{u}_{j} \frac{\partial \varepsilon}{\partial x_{j}}=\frac{\partial}{\partial x_{j}}\left(\frac{v_{t}}{\sigma_{\varepsilon}} \frac{\partial \varepsilon}{\partial x_{j}}\right)+C_{1 \varepsilon} \frac{\varepsilon}{K} v_{t}\left(\frac{\partial \bar{u}_{i}}{\partial \mathrm{x}_{j}}+\frac{\partial \bar{u}_{j}}{\partial x_{i}}\right) \frac{\partial \bar{u}_{i}}{\partial \mathrm{x}_{j}}-C_{2 \varepsilon} \frac{\varepsilon^{2}}{K} ; i, j=1,2,3
\end{gathered}
$$

where, $C_{K} \approx C_{\omega \varepsilon}=0.08-0.09, C_{\omega L} \approx 1.0, \sigma_{K}=1.0, \sigma_{\varepsilon}=1.30, C_{1 \varepsilon}=1.44$ and $C_{2 \varepsilon}=1.92$. The turbulent viscosity is calculated by $v_{t}=C_{\mu} K^{2} / \varepsilon$, where $C_{\mu}=0.09$.

The Wilcox (1993) model is a two-equation $K-\omega$ turbulence closure scheme. The $K$ and $\omega$ equations are determined through (57) and (58):

$$
\begin{gathered}
\frac{\partial K}{\partial t}+\bar{u}_{j} \frac{\partial K}{\partial x_{j}}=\frac{\partial}{\partial x_{j}}\left[\left(v_{l}+C_{1 K} v_{t}\right) \frac{\partial K}{\partial x_{j}}\right]+v_{t}\left(\frac{\partial \bar{u}_{i}}{\partial \mathrm{x}_{\mathrm{j}}}+\frac{\partial \bar{u}_{j}}{\partial x_{i}}\right) \frac{\partial \bar{u}_{i}}{\partial \mathrm{x}_{j}}-C_{2 K} K \omega ; i, j=1,2,3 \\
\frac{\partial \omega}{\partial t}+\bar{u}_{j} \frac{\partial \omega}{\partial x_{j}}=\frac{\partial}{\partial x_{j}}\left[\left(v_{l}+C_{1 \omega} v_{t}\right) \frac{\partial \omega}{\partial x_{j}}\right]+C_{2 \omega} \frac{\omega}{K} v_{t}\left(\frac{\partial \bar{u}_{i}}{\partial \mathrm{x}_{\mathrm{j}}}+\frac{\partial \bar{u}_{j}}{\partial x_{i}}\right) \frac{\partial \bar{u}_{i}}{\partial \mathrm{x}_{j}}-C_{3 \omega} \omega^{2} ; i, j=1,2,3
\end{gathered}
$$

where $C_{1 K}=0.50, C_{2 K}=0.09, C_{1 \omega}=0.50, C_{2 \omega}=5 / 9$ and $C_{3 \omega}=3 / 40$. The turbulent viscosity is calculated by $v_{t}=K / \omega$.

\section{Numerical approaches}

\subsection{DV boundary layer models}

\subsubsection{One- and two-equation models of the K-L type}

Equations system (22) can be easily solved applying an implicit finite-difference approach in the raw unknowns $\left(u, v, K, L, C, v_{t}\right.$, and $\left.\gamma_{t}\right)$ of five differential equations, both in space and time, and two algebraic ones.

Final solution for the vertical profiles of the horizontal components of the velocity $(u, v)$, turbulent kinetic energy $(K)$, macroscale of the eddies $(L)$, concentration $(C)$ and turbulent viscosity $\left(v_{t}\right)$, is obtained iteratively during the time-period $T$ of the signal introduced at the upper limit of the boundary layer. A flowchart representing the numerical solution implemented is presented in figure 3.

\subsubsection{Zero-equation model}

The model equations (29) are to be solved in this section. Considering the $u_{d}$, we note that the non-linear term should be linearized in time using Taylor series. With $u_{t}=l_{m}^{2} \partial u_{d} / \partial z$, the following form of the $u_{d}$-equation show how the solution could be obtained: 


$$
\frac{\partial u_{t}}{\partial t}=\frac{\partial}{\partial z}\left(\left|u_{t}\right| u_{t}\right)=>\left\{\begin{array}{l}
\frac{\partial}{\partial z}\left(u_{t}\right) \text { if } u_{t}>0 \\
-\frac{\partial}{\partial z}\left(u_{t}\right) \text { if } u_{t}<0
\end{array}\right.
$$

Considering the case $u_{d}>0$, a discretized form of this equation reads:

$$
A_{j} u_{d j-1}^{n+1}+B_{j} u_{d j}^{n+1}+C_{j} u_{d j+1}^{n+1}=D_{j} ; 2 \leq j \leq J-1
$$

where the coefficients $A_{j}, B_{j}, C_{j}$ and $D_{j}$ are:

$$
\begin{gathered}
A_{j}=-2 \frac{\Delta t}{\Delta z_{j}}\left(\frac{l_{m j-1 / 2}}{\Delta z_{j-1 / 2}}\right)^{2}\left|\Delta u_{d j-1 / 2}^{\mathrm{n}}\right| ; C_{j}=-2 \frac{\Delta t}{\Delta z_{j}}\left(\frac{l_{m j+1 / 2}}{\Delta z_{j+1 / 2}}\right)^{2}\left|\Delta u_{d j+1 / 2}^{\mathrm{n}}\right| ; \\
B_{j}=1-A_{j}-C_{j} ; D_{j}=u_{d j}^{n}-\frac{1}{2} A_{j} \Delta u_{d j-1 / 2}^{\mathrm{n}}-\frac{1}{2} C_{j} \Delta u_{d j+1 / 2}^{\mathrm{n}}
\end{gathered}
$$

with $\Delta u_{d j-1 / 2}^{\mathrm{n}}=u_{d j}^{\mathrm{n}}-u_{d j-1}^{\mathrm{n}}$ and $\Delta u_{d j+1 / 2}^{\mathrm{n}}=u_{d j+1}^{\mathrm{n}}-u_{d j}^{\mathrm{n}}$.

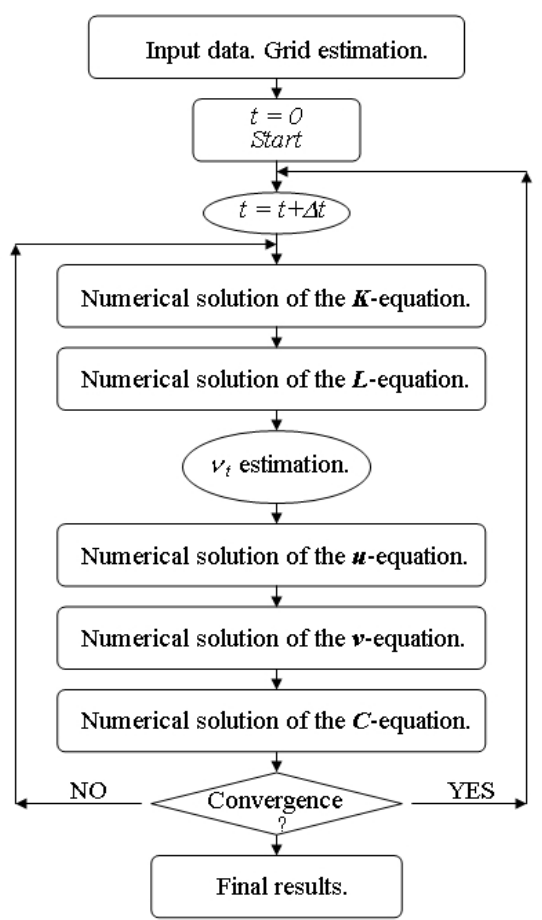

Fig. 3. Flowchart for the 1DV two-equation $K-L$ boundary layer model Applications of 1DV boundary layer models are presented later, in this chapter. 


\subsection{DV boundary layer model}

Equations (50) to (54) are easily solved applying an implicit finite-difference approach centred in space and forward in time. The alternating direction implicit (ADI) method is used to solve the equations for $\xi$ and $K$. The Poisson equation for $\Psi$ is solved by the bloccyclic reduction method (Roache, 1976), which allows a huge saving in calculation time compared to the Gauss-Seidel iteration method (Huynh-Thanh \& Temperville, 1991). Final solution is obtained iteratively during the time-period $T$ of the signal introduced at the upper limit of the boundary layer. A flowchart representing the numerical solution implemented is presented in figure 4.

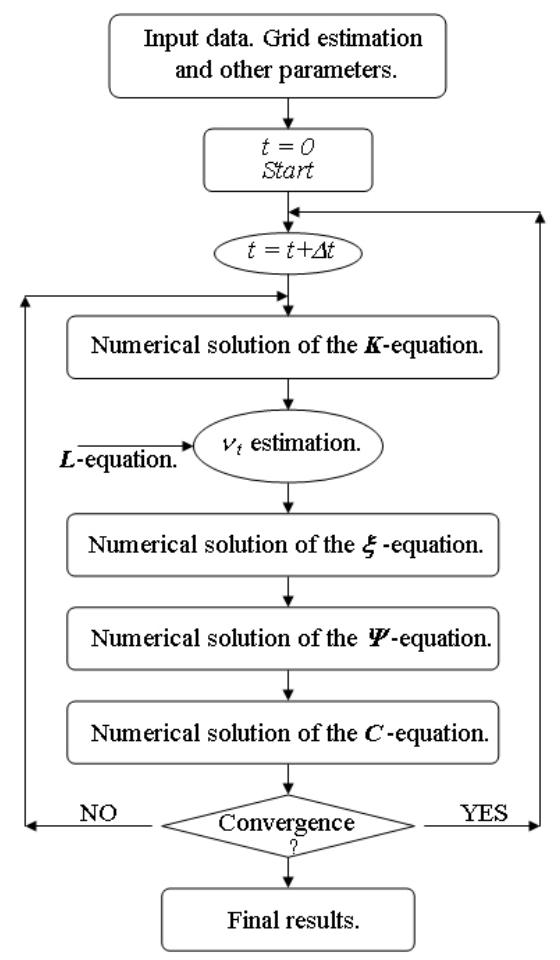

Fig. 4. Flowchart for the 2DV one-equation $K-L$ boundary layer model

Comparisons of laboratory experiments with numerical results of the 2DV boundary layer model are presented later, in section 6.

\section{Parametric formulations}

Following we show how different parametric approaches are derived, and tested with experimental data, using the two-equation $K-L$ boundary layer model (22). Using this model, Huynh Thanh (1990) proposed formula (59) below for the wave friction coefficient, $f_{w(r)}$, in the rough turbulent flow case: 


$$
f_{w(r)}=c_{1} \exp \left[c_{2}\left(\frac{A}{K_{N}}\right)^{n_{1}}\right]
$$

where $A$ is the wave excursion amplitude, and with the empirical coefficients $c_{1}, c_{2}$ and $n_{1}$ determined by Huynh Thanh, and presented in table 1 (formula $\boldsymbol{H T}_{\mathrm{fwr}}$ ). Using the same boundary layer model (22), considering the best overall fit with a large number of the model results, in the interval $6.4 \times 10^{-1} \leq A / k_{N} \leq 3.4 \times 10^{3}$, Antunes do Carmo et al. (2003) proposed formula (59) with the empirical coefficients determined in that study, listed in table 1 as formula $C T_{\mathrm{fwr}}$.

\begin{tabular}{|c|c|c|c|}
\hline $\begin{array}{c}\text { Coeff. } \\
\text { Formula }\end{array}$ & $\boldsymbol{c}_{1}$ & $\boldsymbol{c}_{2}$ & $\boldsymbol{n}_{\boldsymbol{1}}$ \\
\hline $\boldsymbol{H} \boldsymbol{T}_{f w r}$ & 0.00278 & 4.6500 & -0.2200 \\
\hline $\boldsymbol{C} \boldsymbol{T}_{f w r}$ & 0.00140 & 4.5840 & -0.1340 \\
\hline
\end{tabular}

Table 1. Fitting coefficients $c_{1}, c_{2}$ and $n_{1}$, for model of Huynh Thanh (1990) $\left(=\boldsymbol{H} T_{f w r}\right)$ and proposed by Antunes do Carmo et al. (2003) $\left(=C T_{f w r}\right)$

In the case of a current alone, Huynh Thanh found that the friction coefficient $f_{c(r)}$ coincides with the value obtained by the theoretical formula (60):

$$
f_{c(r)}=2\left[\frac{k}{\operatorname{Ln}\left(h / z_{0}\right)-1}\right]^{2}
$$

\subsection{Sinusoidal wave alone}

Considering rough turbulent flows, for values of the wave friction coefficient, $f_{w(r)}$, Antunes do Carmo et al. (2003) proposed formula (59) with $\boldsymbol{C} \boldsymbol{T}_{\text {fwr }}$ coefficients (table 1); Tanaka \& Thu (1994) suggested formula (61), Swart (1974) formula (62) and Soulsby et al. (1994) formula (63):

$$
\begin{gathered}
f_{w(r)}=\exp \left(-7.53+8.07\left(A / z_{0}\right)^{-0.10}\right) \\
f_{w(r)}=0.00251 \exp \left(5.21\left(A / K_{N}\right)^{-0.19}\right) \\
f_{w(r)}=1.39\left(A / z_{0}\right)^{-0.52}
\end{gathered}
$$

A comparison between formulae (59), with $\boldsymbol{H} \boldsymbol{T}_{\mathrm{fwr}}$ and $\boldsymbol{C} \boldsymbol{T}_{\mathrm{fwr}}$ coefficients, (61), (62) and (63) is shown in Antunes do Carmo et al. (2003). The same figure also shows experimental measurements of Sleath (1987), Kamphuis (1975), Jensen et al. (1989), Sumer et al. (1987) and Jonsson \& Carlsen (1976).

According to Sleath (1991), bottom shear stress may be split into two components:

$$
\hat{\tau}_{w p}=\hat{\tau}_{w}+\hat{\tau}_{p}
$$


The shear stress in the fluid, $\hat{\tau}_{w}$, is taken into account by the model, but the value of $\hat{\tau}_{p}$, due to the mean pressure gradient acting on the bed roughness, is not. Using Sleath's experiments, it can be seen that a global friction coefficient may be split into the following two components:

$$
f_{w p}=f_{w}+f_{p}
$$

where $f_{w}$ represents the friction coefficient obtained by the $K-L$ model, and $f_{p}$ represents the pressure gradient contribution. Assuming $K_{N}=2.5 d_{50}$, Sleath (1991) presented the formula (66):

$$
f_{p}=0.48\left(A / K_{N}\right)^{-1}
$$

The pressure gradient was not taken into account in experiments conducted by Sleath, Sumer, Jensen and Jonsson. Therefore, results of their experimental data are compared with model (59) considering $\mathrm{CT}_{\mathrm{fwr}}$ coefficients. Excluding a small part of the Sleath's experiments, all other cases show a close agreement model (Antunes do Carmo et al., 2003). Discrepancies are explained as a consequence of some of Sleath's experiments being in the smooth-laminar transition regime. The pressure gradient is taken into account in Kamphuis' experiments, so this data should be compared with values for the following expression (67):

$$
f_{w p}=f_{w}+f_{p}=0.0014 \exp \left[4.584\left(\frac{A}{K_{N}}\right)^{-0.134}\right]+0.48\left(\frac{A}{K_{N}}\right)^{-1}
$$

For values of $A / K_{N}>100$, the $f_{p}$ term is negligible and expression (59) with $C \boldsymbol{T}_{\text {fwr }}$ coefficients (table 1) is in close agreement with results (Antunes dio Carmo et al., 2003).

\subsection{Time-dependent shear stress}

For the purpose of calculating time-dependent shear stress $\tau(t)$ in the case of an irregular wave whose instantaneous velocity is given by $U(t)$, Soulsby et al. (1994) propose calculating the value of the friction coefficient $f_{w}$ for the equivalent sinusoidal wave with orbital velocity amplitude equal to $\sqrt{2} U_{r m s}$ and period $T_{p}$. It can therefore be deduced (Antunes do Carmo et al., 2003):

$$
f_{w}=1.39\left(\frac{A}{z_{0}}\right)^{-0.52} \quad A=\frac{\sqrt{2} U_{r m s}}{2 \pi}
$$

where $U_{r m s}=$ root-mean-square of orbital velocities. For a sinusoidal wave, this formulation correctly represents, in parametric form, the bottom shear stress obtained using $K-L$ model (22), but does not take into account the phase shift between $\tau(t)$ and $U(t)$. For an asymmetric wave, or an irregular wave, more important differences appear between this parametric formulation and the results calculated directly by the $K-L$ model.

To illustrate these phenomena, we consider the instantaneous velocity records presented in figure 5 for three cases (Antunes do Carmo et al., 2003): a) a sinusoidal wave, with orbital velocity amplitude $0.225 \mathrm{~m} / \mathrm{s}$ and period $3.6 \mathrm{sec}$; b) a cnoidal wave, with a total velocity amplitude $1.107 \mathrm{~m} / \mathrm{s}$ and period $9 \mathrm{sec}$, and c) an irregular wave obtained by the non-linear 
propagation of a sinusoidal wave, making use of a numerical Boussinesq-type model (Antunes do Carmo et al., 1993), with a $3.0 \mathrm{sec}$ period in a channel $0.30 \mathrm{~m}$ depth.

The values of the friction coefficient for a sinusoidal wave are shown in figure 6 . Close agreement is evident between results 1 and 2. The instantaneous bottom shear stresses $\tau(t)$ have been calculated using model (59) with $\boldsymbol{C T}_{\text {fwr }}$ coefficients. In figure 7 , results given by the $K-L$ model (22) (result 2) are compared both with those of model (59) (result 1) and with those obtained by a constant friction coefficient without the phase shift (result 3 ). Computed shear stresses for the sinusoidal wave case are presented in figure 7-a). Results of the model (59) with $C \boldsymbol{T}_{\text {fwr }}$ coefficients (result 1) are in close agreement with those of the $K-L$ model (22).
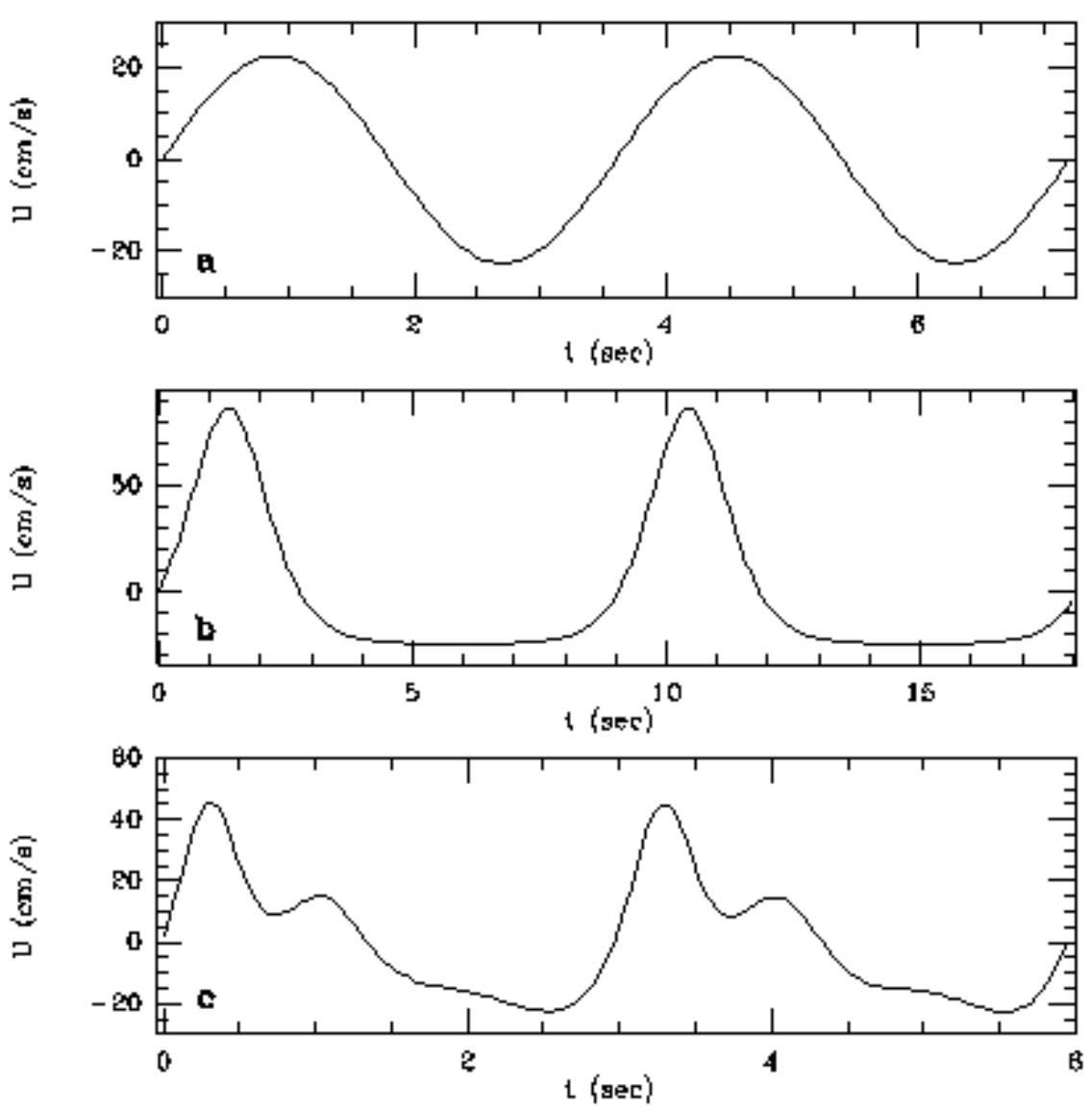

Fig. 5. Instantaneous velocity records: a - Sinusoidal wave (orbital velocity amplitude = $0.225 \mathrm{~m} / \mathrm{s}$, period $=3.6 \mathrm{sec}$ ); $\mathrm{b}-$ Cnoidal wave (total velocity amplitude $=1.107 \mathrm{~m} / \mathrm{s}$, period $=9.0 \mathrm{sec}$ ); $\mathrm{c}$ - Irregular wave (resulting from the non-linear propagation of a sinusoidal wave with a period $=3.0 \mathrm{sec}$ in a channel $0.30 \mathrm{~m}$ depth) (Antunes do Carmo et al., 2003) 


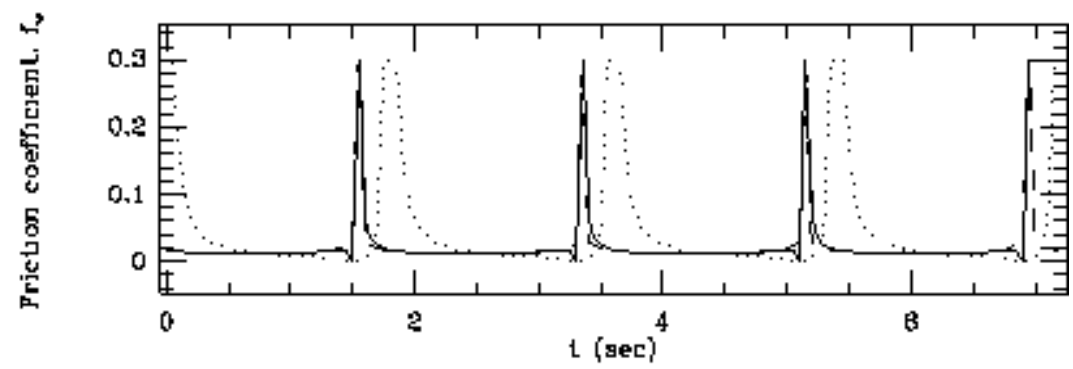

Fig. 6. Comparisons between the parameterized friction coefficient and the $K-L$ model result for a sinusoidal wave. Model (59) with $\boldsymbol{C} \boldsymbol{T}_{\text {fwr }}$ coefficients (result 1: - - - ; result 3: ........) and that obtained by $K-L$ model (result 2: - (Antunes do Carmo et al., 2003)
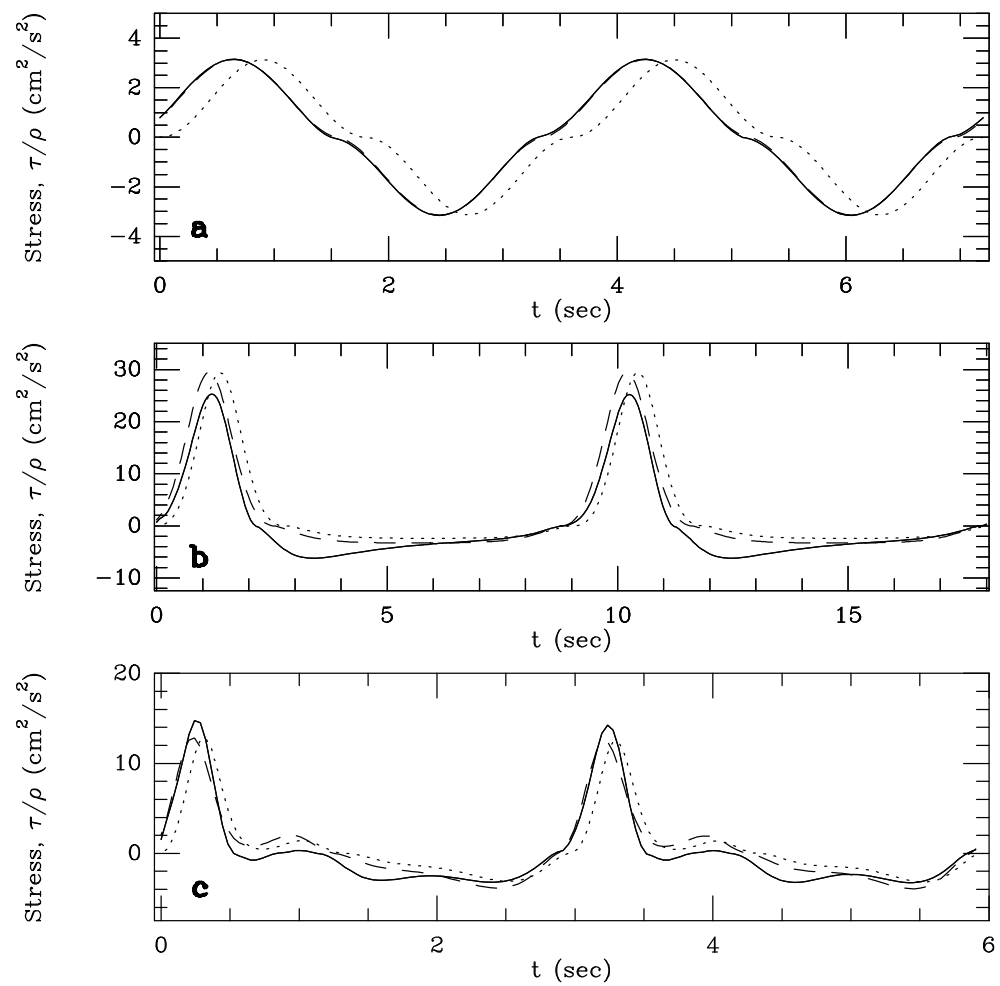

Fig. 7. Comparisons between the parameterized shear stress and the $K-L$ model result: a) Sinusoidal wave; b) Cnoidal wave; c) Irregular wave. Model (59) with $\boldsymbol{C T}_{\text {fwr }}$ coefficients (result 1: ---- ; result 3: …...) and that obtained with $K-L$ model (result 2: - ) (Antunes do Carmo et al., 2003)

A phase error between result 3 and result 2 ( $K-L$ model) is evident. In the cnoidal wave case, the bottom shear stress calculated by the numerical boundary layer model is represented in figure 7-b) by the continuous line. As can be seen, for this case (figure 7-b), 
result 1 is closer to result 2 than it is to result 3, for both phase and negative values. However, asymmetries are not reproduced and a discrepancy can be seen for the maximum value. Several observations can be made concerning these results (Antunes do Carmo et al., 2003): $i$ ) The representative curve $\tau(t)$ does not present the symmetry of velocities $U(t)$. The negative values of $\tau(t)$ are more important after the main positive peak than before it. It may be assumed that a "turbulence memory" created for this main peak influences what happens afterwards; ii) If the maximum velocity value is considered to be $U_{1}$ and the minimum velocity value $U_{2}$, it follows that:

$$
\frac{\tau_{2}}{\tau_{1}}=\left(\frac{U_{2}}{U_{1}}\right)^{2}=0.08
$$

Figure 7-b) shows that the relation $\tau_{2} / \tau_{1}=0.24$ is greater than the value calculated by (69). Therefore, as in the case of a sinusoidal wave, the friction coefficient does not remain constant when velocity changes, assuming increasing values with decreasing velocity. Antunes do Carmo et al. (2003) propose calculating a time-dependent friction coefficient by replacing the maximum velocity with the instantaneous velocity $U(t+\theta)$, which takes into account the phase shift. The coefficient $f(t)$ will accordingly be calculated using expression (59), with $C T_{\text {fwr }}$ coefficients (table 1 ), where $A$ is given by (70):

$$
A=\frac{\sqrt{2} U_{r m s} T_{p}}{2 \pi} \frac{U(t+\theta)}{U_{\max }}
$$

and $\tau(t)$ is defined by (71):

$$
\tau(t)=\frac{f(t)}{2} U(t+\theta)|U(t+\theta)|
$$

$\theta$ represents the phase lag between $U(t)$ and the bottom shear stress $\tau(t)$ at the upper limit of the boundary layer. Computed shear stresses for the more complex velocity case (irregular wave obtained by the non-linear propagation of an input sinusoidal wave) is presented in figure 7-c). A comparison of results 1 and 3 with result 2 shows that result 1 is still closer to that of the $K-L$ model (22) than to result 3. Also, a slight discrepancy can be seen for the maximum value. Despite the "turbulence memory effects", the model (59) with $C \boldsymbol{T}_{\text {fwr }}$ coefficients fits closely with the boundary layer model results for the three cases analysed. Comparisons were made, however, assuming that results given by the $K-L$ model correctly represent the real conditions. Moreover, some discrepancies occur, especially for the maximum values.

\section{Applications}

\subsection{K-L 1DV boundary layer model}

Following closely Antunes do Carmo et al. (1996), an application of the $K-L$ turbulence model is presented, which corresponds to a sinusoidal mass oscillation where the velocity at the top of the bottom boundary layer is a pure sinusoidal wave with amplitude $\bar{u}=170$ $\mathrm{cm} / \mathrm{s}$ and period $7.2 \mathrm{sec}$. The following values were considered: $w_{\mathrm{s}}=2.6 \mathrm{~cm} / \mathrm{s}, d_{50}=0.021$ $\mathrm{cm}, z_{0}=0.175 \times 10^{-2} \mathrm{~cm}, z_{a}=2 d_{50}=0.042 \mathrm{~cm}$ and $z_{\delta}=16.2 \mathrm{~cm}$. Figure 8-a) to d) show the 
time series of sediment concentration computed at different levels above $z_{a}(\mathrm{z}=0.10,1.62$, 2.08 and $4.54 \mathrm{~cm}$ ). In figure 8-e) the vertical profiles of sediment concentration with phase shift of $60^{\circ}$ are plotted (full lines), as well as the mean values over a wave period (dash lines). In each case the numerical solutions are compared to experimental data obtained by Ribberink \& Al-Salem (Tran-Thu, 1995). Finally, in figure 8-f) the eddy diffusivity vertical profile averaged over a wave period is plotted.

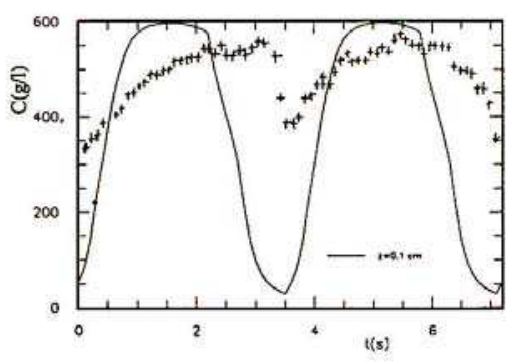

a) Sediment concentration at $z=0.10 \mathrm{~cm}$.

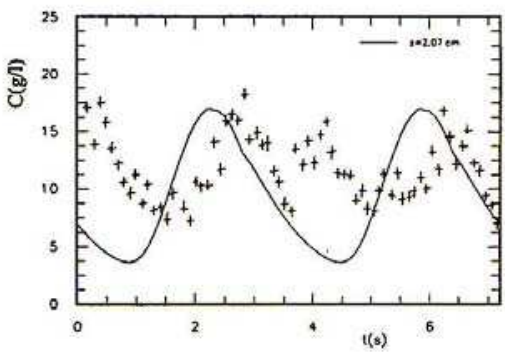

c) Sediment concentration at $\mathrm{z}=2.08 \mathrm{~cm}$.

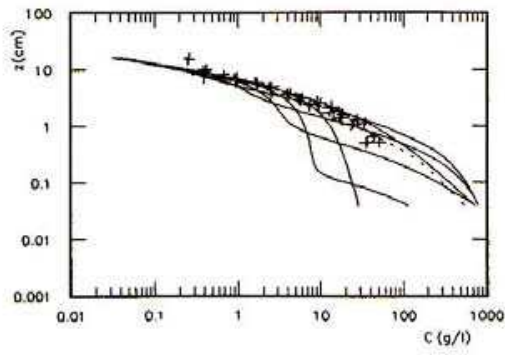

e) Vertical profile of sediment cncentration with phase shift of $60^{\circ}$.

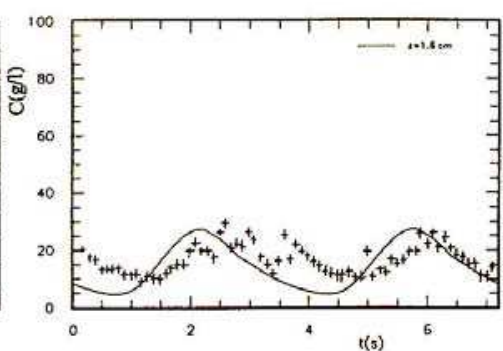

b) Sediment concentration at $\mathrm{z}=1.62 \mathrm{~cm}$.

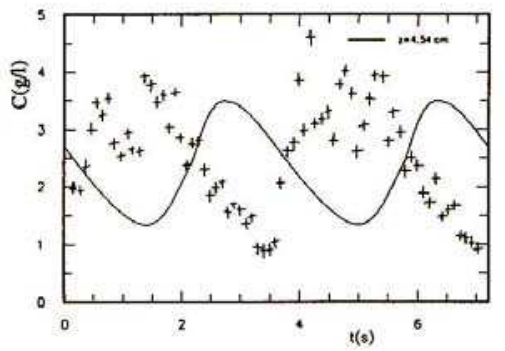

d) Sediment concentration at $\mathrm{z}=4.54 \mathrm{~cm}$.

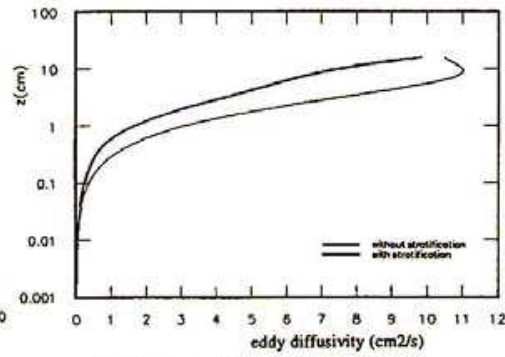

f) Eddy diffusivity vertical profile averaged over a wave period.

Fig. 8. Sinusoidal mass oscillation (Antunes do Carmo et al., 1996) 
The analyses of results show that:

i. the vertical distribution of sediment agrees well with experimental data;

ii. the pick concentration in the time series occurs with larger and larger phase the further away the level is located from the bed;

iii. at the upper levels, a time phase shift between the computed values of concentration and the experimental ones is observed;

iv. in the vicinity of the bottom (figure 8-a)) the time series of concentration shows the intermittence phenomena;

v. the maximum values of sediment concentration agree well with data at all levels.

\subsection{DV boundary layer model}

The flow in the bottom boundary layer established over a rippled bed was investigated through experiments and numerical calculations with a 2DV model. Experiments were conducted in an oscillatory flow tunnel illustrated in figure 9. This device was built from an existing wave flume at the Department of Civil Engineering of the University of Coimbra, Portugal. The wave tunnel has a rectangular cross section with $0.30 \mathrm{~m}$ width and $0.20 \mathrm{~m}$ high. The total length of the tunnel is $7.5 \mathrm{~m}$.

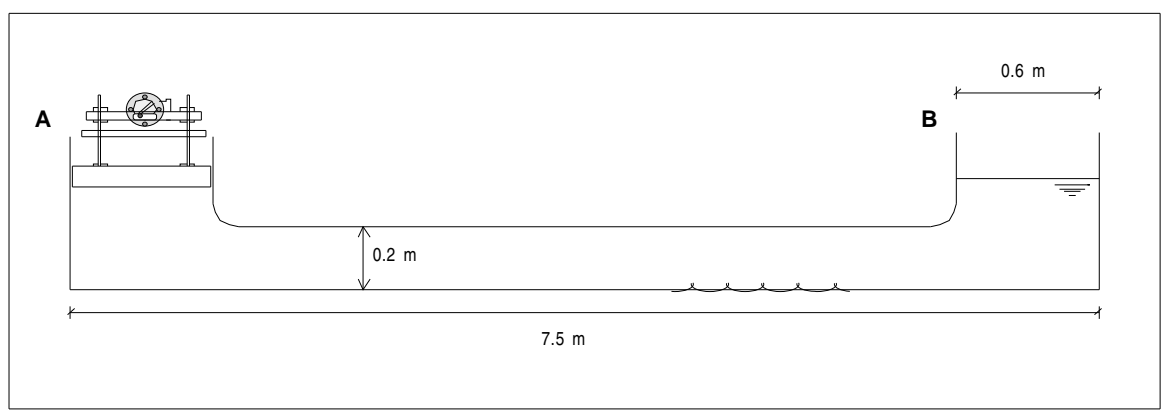

Fig. 9. Wave tunnel

At the left end (A) the vertical motion of a wave paddle produces an oscillatory flow within the tunnel. Five artificial symmetrical ripples have been placed on the tunnel's bed: each of the ripples has a length $\left(L_{r}\right)$ of $7 \mathrm{~cm}$ and height $\left(H_{r}\right)$ equal to $1.2 \mathrm{~cm}$. The ripples were made in aluminium with the following profile (72):

$$
z=\frac{4 H_{r}}{L_{r}{ }^{2}} x^{2}-\frac{4 H_{r}}{L_{r}} x+H_{r} ; 0 \leq x \leq L_{r} / 2
$$

Sediment with a median grain diameter of $0.27 \mathrm{~mm}$ was glue to the surface of the ripples in order to simulate the skin roughness. Velocities were measured with an acoustic Doppler system (ADV) under sinusoidal oscillations at the wave paddle, over one ripple crest and one trough. Table 2 presents the experimental conditions considered in one of the tests made, being $z_{1}$ the height above the crest where the measurements were done. With the configuration of the ADV used, the measurements could only be done for heights above 4 $\mathrm{cm}$ from the bed. Figure 10 represents the mean values of the measured values of $u$ and $w$ at different levels during the wave cycle: $u$ and $w$ represent, respectively, the horizontal velocity in wave's tunnel direction and the vertical velocity. 


\begin{tabular}{|c|c|c|c|c|}
\hline Serie & $\mathbf{N r}$ & Crest/Trough & $\mathbf{T} \mathbf{( s )}$ & $\mathbf{Z}_{\mathbf{i}}(\mathbf{c m})$ \\
\hline S1 & 1 & $\mathrm{Cr}$ & 3.60 & 3.9 \\
\hline- & 2 & $\mathrm{Cr}$ & 3.60 & 4.0 \\
\hline- & 3 & $\mathrm{Cr}$ & 3.60 & 4.8 \\
\hline- & 4 & $\mathrm{Cr}$ & 3.60 & 5.7 \\
\hline- & 5 & $\mathrm{Cr}$ & 3.60 & 6.8 \\
\hline- & 6 & $\mathrm{Cr}$ & 3.60 & 7.9 \\
\hline- & 7 & $\mathrm{Cr}$ & 3.60 & 8.9 \\
\hline- & 8 & $\mathrm{~T}$ & 3.60 & 2.6 \\
\hline- & 9 & $\mathrm{~T}$ & 3.60 & 2.8 \\
\hline- & 10 & $\mathrm{~T}$ & 3.60 & 3.1 \\
\hline- & 11 & $\mathrm{~T}$ & 3.60 & 3.6 \\
\hline- & 12 & $\mathrm{~T}$ & 3.60 & 4.1 \\
\hline- & 13 & $\mathrm{~T}$ & 3.60 & 4.6 \\
\hline- & 14 & $\mathrm{~T}$ & 3.60 & 5.1 \\
\hline- & 15 & $\mathrm{~T}$ & 3.60 & 6.1 \\
\hline- & 16 & $\mathrm{~T}$ & 3.60 & 8.1 \\
\hline- & 17 & $\mathrm{~T}$ & 3.60 & 9.9 \\
\hline
\end{tabular}

Table 2. Experimental conditions

$u / U_{w}$

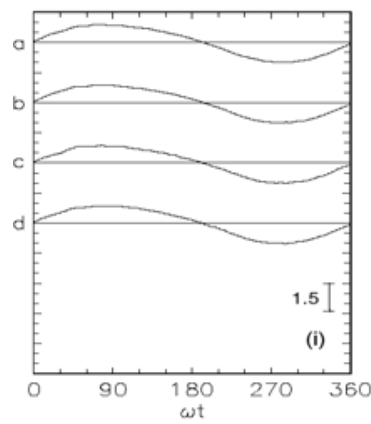

$u / U_{w}$

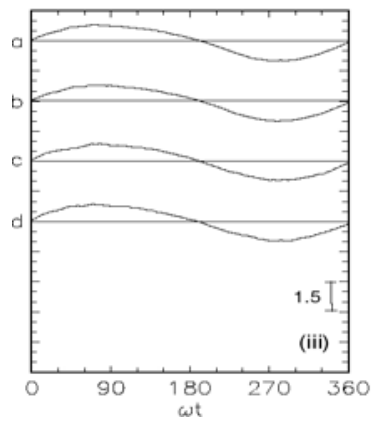

$\mathrm{w} / \mathrm{U}_{\mathrm{w}}$

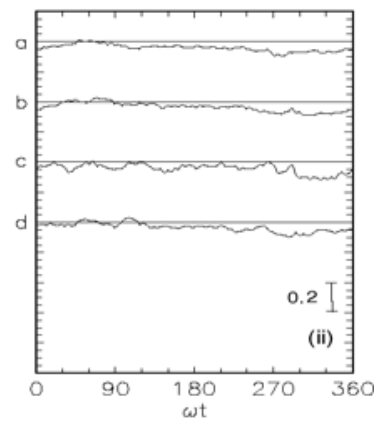

$w / U_{w}$

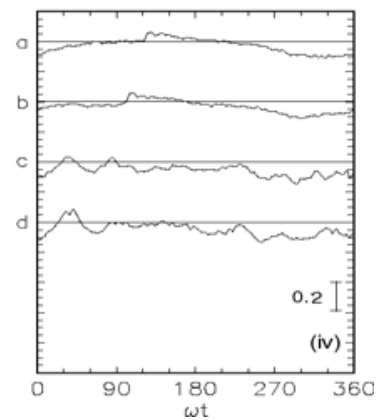

Fig. 10. Measured time series of $U$ and $W$ for different levels above the crest (i, ii) - ( $z_{1}(a)=$ $\left.8.9 \mathrm{~cm}, \mathrm{z}_{1}(\mathrm{~b})=7.9 \mathrm{~cm}, \mathrm{z}_{1}(\mathrm{c})=4.0 \mathrm{~cm}, \mathrm{z}_{1}(\mathrm{~d})=3.9 \mathrm{~cm}\right)$ and above the trough (iii, iv) $-\left(\mathrm{z}_{1}(\mathrm{a})=\right.$ $\left.9.9 \mathrm{~cm}, \mathrm{z}_{1}(\mathrm{~b})=8.1 \mathrm{~cm}, \mathrm{z}_{1}(\mathrm{c})=2.8 \mathrm{~cm}, \mathrm{z}_{1}(\mathrm{~d})=2.6 \mathrm{~cm}\right)($ Silva, 2001) 
These values were divided by the amplitude of the horizontal velocity outside the boundary layer, $U_{w}$, and were obtained by averaging equi-phase data over approximately 20 wave periods. The analysis of figure 10 shows that: (1) The oscillatory flow in the wave tunnel does not correspond to a sinusoidal oscillation as we can observe from the velocities measured at the highest levels (a), and (2) At the lower levels (c, d) the measured values of $w$ show oscillations with a time scale inferior to the ones observed in the highest levels: this suggest that the flow at those levels is perturbed by the lee vortex developed during the wave cycle and that are ejected from the bottom after flow reversal $\left(0^{\circ}\right.$ and $\left.180^{\circ}\right)$.

A numerical simulation of the flow in the bottom boundary layer was done with the 2DV model (50) to (54). In figure 11 the numerical results are compared with the experimental data. The results are only plotted for the lower level of measurements.
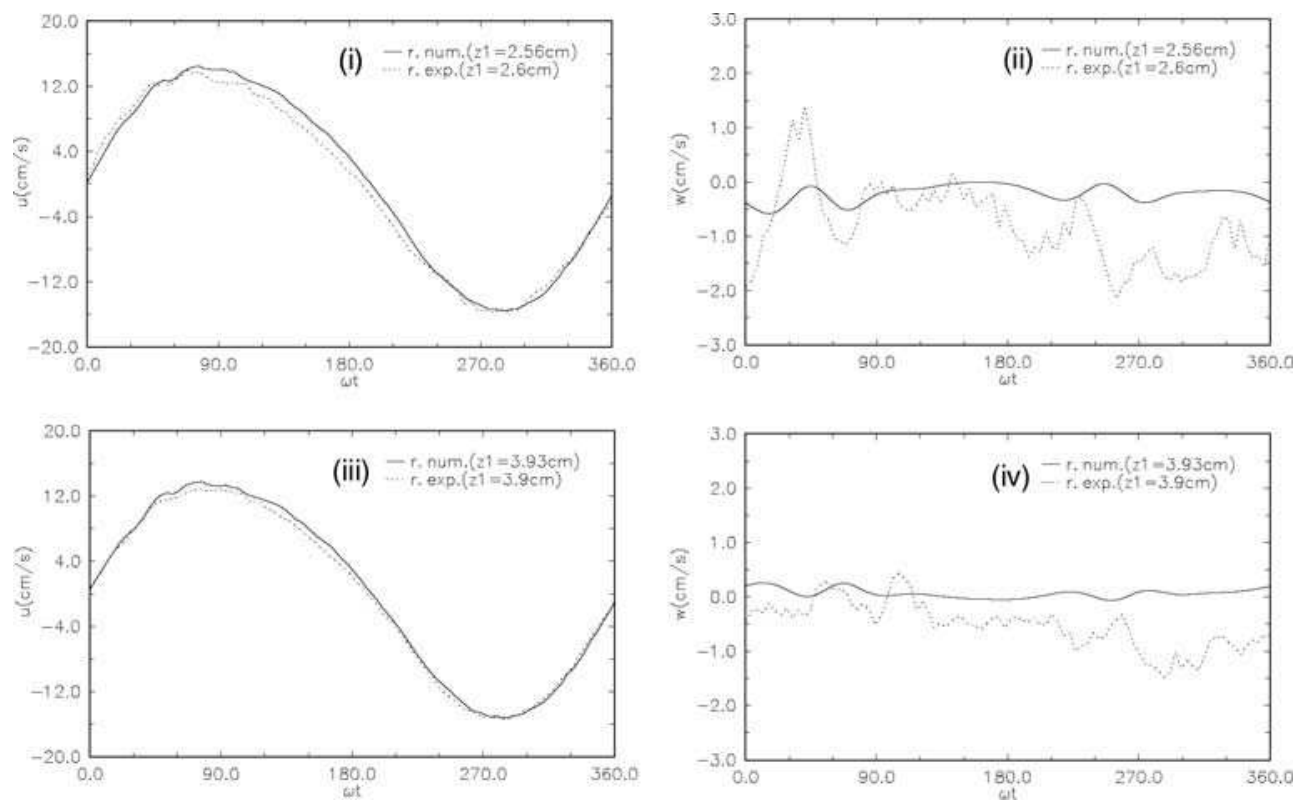

Fig. 11. Numerical results vs experimental data (Silva, 2001)

It is seen that there is a good agreement between the computed and measured horizontal velocity. The computed vertical velocity shows small oscillations after flow reversal, between $0^{\circ}-90^{\circ}$ and $210^{\circ}-300^{\circ}$. The shape of these oscillations is similar to the observed one, although there is a phase shift between them. The amplitude of these oscillations is also lower than the amplitude of the measured values of $w$ : this means that the model dissipates the kinetic energy of the ejected vortex at a rate that is superior to what it is observed. This feature has also been noted in other comparisons. To analyse with more detail the flow in the bottom boundary layer, namely the vortex paths during the wave cycle, we have plotted in figure 12 the vorticity field at different wave phases. It is seen that the lowest level of measurements over the ripple crest is above the track of the vortex that is carried by the flow after it is ejected. This justifies the poor agreement between the numerical and experimental results in the figure 11 (iv). 

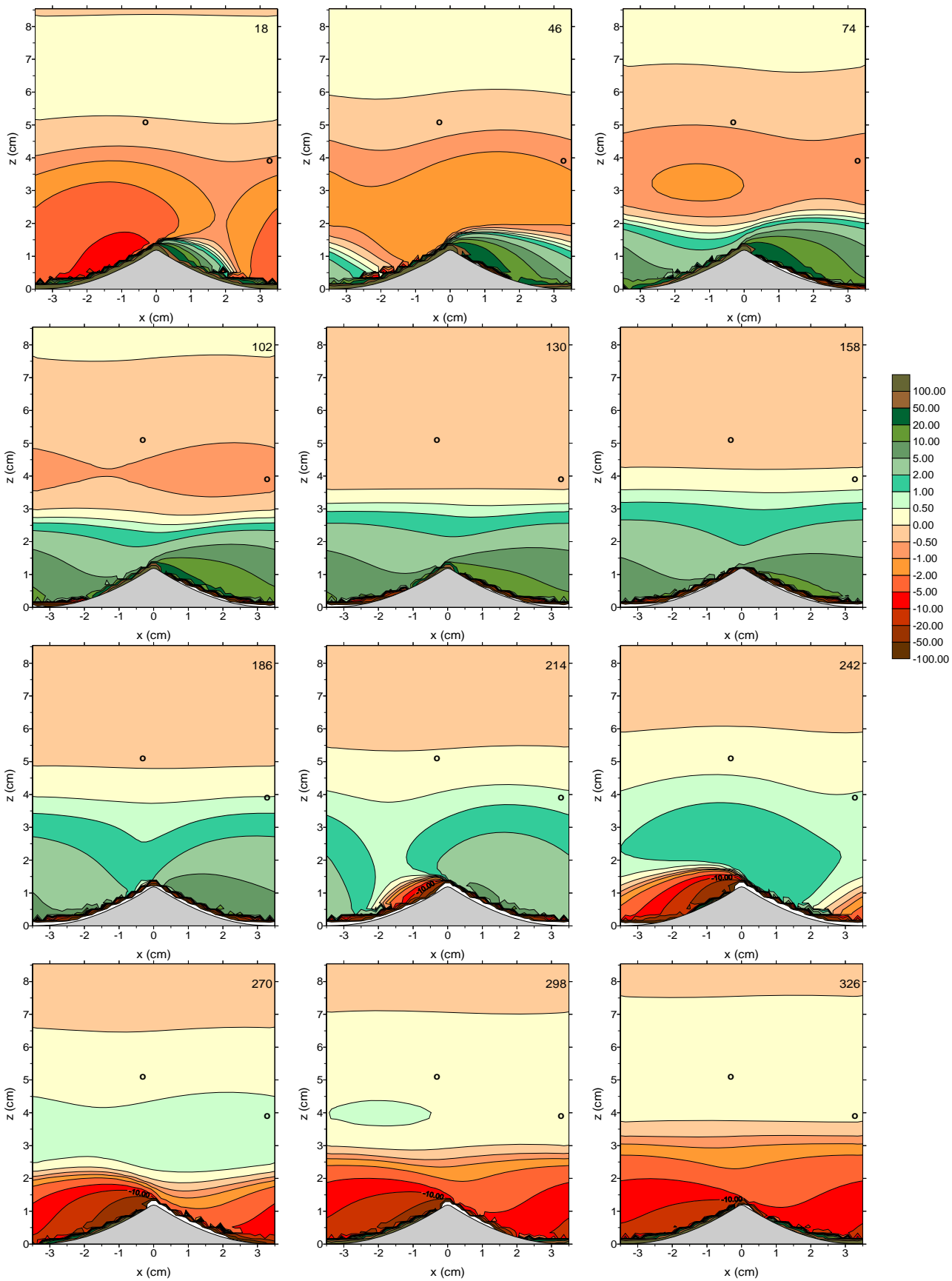

Fig. 12. Computed vorticity field $\left(s^{-1}\right)$ at different phases of the flow. The lower levels of the measurements over the ripple crest and trough are marked $\bullet$ 


\section{Conclusion}

Assuming that the fluid is in a randomly unsteady turbulent state and applying time averaging to the basic equations of motion, the fundamental equations of incompressible turbulent motion are obtained. A three-dimensional form of conservation equations for a single Reynolds stress and for the turbulent kinetic energy is derived. However, as the full three-dimensional form of equations is very complex and not easy to solve, with many unknown correlations to model, other much simpler one- and two-dimensional boundary layer forms of these relations are derived. A brief discussion about numerical models based on control volumes and finite difference approximations is presented to solve 1DV versions of the one- and two-equation rough turbulent bottom boundary layer model of the K-L type, and of the 2DV boundary layer model. These numerical models are then used to calibrate general parametric formulations for the instantaneous bottom shear stress due to both a wave and a wave-current interaction cases. They are still used to discuss some important aspects, like the phase shift and the turbulence memory effects. Mathematical formulations and parametric approaches are extended to include the effect of suspended non cohesive sediments. Comparisons with experimental results show that both 1DV and 2DV boundary layer models are able to predict quite well the complex flow properties. However, these models are strictly valid for permanent flows in the fully developed turbulent regime at high Reynolds numbers. When the flow is oscillatory, the condition of local equilibrium of the turbulence is no longer completely satisfied, particularly at the time when the velocity of the potential flow is small. Therefore, improvements are necessary to obtain more precise results for moderate Reynolds numbers.

\section{References}

Aldama, A.A., 1990. Filtering techniques for turbulent flows simulation, Lecture Notes in Engineering, Ed. C.A. Brebbia and S.A. Orszag, 56, Springer-Verlag, ISBN 3-54052137-2.

Antunes do Carmo, J.S., Seabra-Santos, F.J. \& Barthélemy, E., 1993. Surface waves propagation in shallow-water: a finite element model, Int. J. Num. Meth. in Fluids, Vol. 16, No. 6, 447-459.

Antunes do Carmo J.S., Silva P. \& Seabra-Santos F.J., 1996. The K-L turbulence model vs parametric formulations, Hydraulic Engineering Software VI, 323-334, Ed. W.R. Blain, WITPRESS/CMP. ISBN 1-85312-405-2.

Antunes do Carmo, J.S., Temperville, A. \& Seabra-Santos F.J., 2003. Bottom friction and time-dependent shear stress for wave-current interaction, Journal of Hydraulic Research, IAHR, Vol. 41, No 1, 27-37.

Batchelor, G.K., 1953. The Theory of Homogeneous Turbulence. Cambridge, UK: Cambridge, Univ. Press.

Boussinesq, 1877. Essai sur la théorie des eaux courantes. Institute de France, Académie des Sciences, Memoires présentées par divers savants, Vol. 23, No. 1 (in French).

Chen, C.J., Shih, C., Lienau, J. \& Kung, R.J., 1996. Flow modeling and turbulence measurements, A.A. Balkema, Rotterdam / Brookfield, ISBN: 9054108266. 
Fourniotis, N.Th., Dimas, A.A \& Demetracopoulos, A.C., 2006. Spatial development of turbulent open-channel flow over bottom with multiple consecutive dunes. Proceedings of the International Conference River Flow 2006, Lisbon, Portugal, 10231032.

Hinze, J., 1975. Turbulence (2nd Edition), McGraw-Hill Classic Textbook Reissue Series.

Huynh-Thanh, S., 1990. Modélisation de la couche limite turbulente oscillatoire générée par l'interaction houle-courant en zone cotière, Ph.D thesis, INP - Grenoble, France (in French).

Huynh-Thanh, S. \& A. Temperville, A.,1991. A numerical model of the rough turbulent boundary layer in combined wave and current interaction. In: R.L. Soulsby and R. Betess (Editors), Sand Transport in Rivers, Estuaries and the Sea, Balkema, Rotterdam, 93-100.

Jensen, B.L., Sumer, B.M. \& Fredsøe, J., 1989. Turbulent oscillatory boundary layers at high Reynolds numbers, Journal of Fluid Mechanics, 206, 265-297.

Jonsson, I.J. \& Carlsen, N.A., 1976. Experimental and theoretical investigations in an oscillaory turbulent boundary layer, Journal of Hydraulics Research, 14(1), 45-60.

Kamphuis, J.W., 1975. Friction factor under oscillatory waves, J. Waterw. Port Coastal Ocean Eng., 101 (WW2), 135-144.

Kaneda, Y. \& Ishihara, T., 2006. High-resolution direct numerical simulation of turbulence, Journal of Turbulence, Vol. 7, No. 20.

Kolmogorov, A.N., 1941. The local structure of turbulence in incompressible viscous fluid for very large Reynolds numbers. Proceedings of the USSR Academy of Sciences 30: 299-303. (Russian), translated into English by Kolmogorov, Andrey Nikolaevich (July 8, 1991). The local structure of turbulence in incompressible viscous fluid for very large Reynolds numbers. Proceedings of the Royal Society of London, Series A: Mathematical and Physical Sciences 434 (1991): 9-13.

Launder, B.E. \& Spalding, D.B., 1972. Lectures in mathematical models of turbulence, Academic Press London and New York, ISBN:0-12-438050-6.

Lesieur, M., 1997. Turbulence in fluids: Third revised and enlarged edition, Fluid Mechanics and its Applications, Vol. 40, Kluwert Academic Publishers, ISBN 0-7923-4415-4.

Lewellen, W.S., 1977. Use of the invariant modelling, in Handbook of turbulence, Plenum Publishing Corp., Vol. 1, 237-280.

Lumley, J.L., 1996. Fundamental aspects of incompressible and compressible turbulent flows, Simulation and Modeling of Turbulent Flows, Cap. 1. ICASE/LaRC Series in Computacional Science and Engineering. Edited by Gatski, Hussaini e Lumley.

McComb, W.D.: http://www.ph.ed.ac.uk/acoustics/turbulence/ (last acces May 2011).

Mohammadi, B. \& Pironneau, 1994. Analysis of the k-epsilon turbulence model, John Wiley e Sons, ISSN:0298-3168.

Monin, A.S. \& Yaglom, A.M., 1971. Statistical Fluid Mechanics: Mechanics of Turbulence, Vol. 1, The MIT Press, Edited by John L. Lumley, ISBN: 0-262-13062-9.

Moser, R.D., 2006. On the validity of the continuum approximation in high Reynolds number turbulence, Phys. Fluids 18, 078105.

Moulden, T.H., W. Frost \& Garner, A.H., 1978. The complexity of turbulent fluid motion, Handbook of turbulence, W. Frost and T.H. Moulden, Eds, Plenum Press 
Nezu, I.E. \& Nakagawa, H., 1993. Turbulence in open-channel flows. IARH/AIRH Monograph Series, $3^{\text {a }}$ Ed. Balkema.

Prandtl, L., 1925. Über die ausgebildete Turbulenz. Z. Angew, Math. Mech., Vol. 5, 136-139.

Reynolds, O., 1883. On the experimental investigation of the circumstances which determine whether the motion of water shall be direct or sinuous, and the law of resistance in parallel channels Phil. Trans. Roy. Soc. London Ser. A, vol. 174, 935982.

Reynolds, O., 1895. On the dynamical theory of incompressible viscous fluids and the determination of the criterion, Phil. Trans. Roy. Soc. London Ser. A, vol. 186, 123164.

Roache, P.J., 1976. Computational Fluid Dynamics. Eds. Hermosa Publishers, New Mexico.

Rodi, W., 1980. Turbulence Models and their Application in Hydraulics - A State-of-the-Art Review, I.A.H.R - Publication.

Rodi, W., 1984. Turbulent models and their applications in Hydraulics - A state of the art review, 2nd Edition, Bookfield Publishing, 1-36.

Rodi, W., 1993. Turbulence models and their application in hHydraulics, Balkema.

Rotta, J.C., 1951. Statistische theorie nichthamagener turbulenz, Zeitschrift f. Physik, Bd. 192, pp. 547-572, and Bd. 131, 51-77.

Sato, S., Mimura, N. \& Watanabe, A., 1984. Oscilatory boundary layer flow over rippled beds. Proc 19th Conf. Coastal Engineering, 2293-2309.

Sheng, Y.P., 1984. A turbulent transport model of coastal processes. Proc. 19th Conf. Coastal Eng., 2380-2396.

Schiestel, R., 1993. Modélisation et simulation des écoulements turbulents, Hermes, Paris, ISBN: 2-86601-371-9.

Silva, P.M.C.A., 2001. Contribution for the study of sedimentary dynamics in coastal regions, Ph.D thesis, University of Aveiro, Portugal (in Portuguese).

Sleath, J.F.A., 1984. Sea bed Mechanics. Eds. Wiley-Interscience.

Sleath, J.F.A., 1987. Turbulent oscillatory flow over rough beds, Journal of Fluid Mechanics, 182, 369-409.

Sleath, J.F.A., 1991. Velocities and shear stresses in wave-current flows, Journal of Geophysical Research, Vol. 96, No. C8, 15, 237-15, 244.

Soulsby, R.L., Hamm, L., Klopman, G., Myrhaug, D., Simons, R.R. \& Thomas, G.P., 1994. Wave-current interaction within and outside the bottom boundary layer, Coastal Eng., 21, 41-69.

Sumer, B.M., Jensen, B.L. \& L. Fredsøe, L., 1987. Turbulence in oscillatory boundary layers. In Advances in Turbulence, Springer, Heidelberg, 556-567.

Swart, D.H., 1974. Offshore sediment transport and equilibrium beach profiles. Delft Hydraulics Lab., Publ. 131.

Tanaka, H. \& Thu, A., 1994. Full-range equation of friction coefficient and phase difference in a wave-current boundary layer, Coastal Eng., 22, 237-254.

Tennekes, H. \& Lumley, J.L., 1972. A first course in turbulence, MIT Press.

Tran-Thu, T., 1995. Modélisation numérique de l'interaction houle-courant-sédiment, Ph.D thesis, Université Joseph Fourier - Grenoble, France (in French). 
Tran-Thu, T. \& Temperville, A., 1994. Numerical model of sediment transport in the wavecurrent interaction, in Modelling of Coastal and Estuarine Processes, Eds. F. SeabraSantos \& A. Temperville, 271-281.

Wilcox, D.C., 1993. Turbulent modelling of CFD. DCW Industries, La Canada, Calif. 


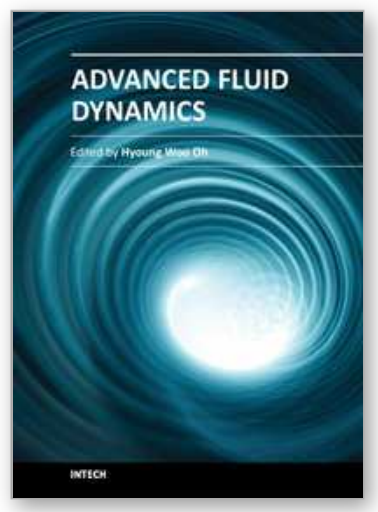

\author{
Advanced Fluid Dynamics \\ Edited by Prof. Hyoung Woo Oh
}

ISBN 978-953-51-0270-0

Hard cover, 272 pages

Publisher InTech

Published online 09, March, 2012

Published in print edition March, 2012

This book provides a broad range of topics on fluid dynamics for advanced scientists and professional researchers. The text helps readers develop their own skills to analyze fluid dynamics phenomena encountered in professional engineering by reviewing diverse informative chapters herein.

\title{
How to reference
}

In order to correctly reference this scholarly work, feel free to copy and paste the following:

José Simão Antunes do Carmo (2012). Turbulent Boundary Layer Models: Theory and Applications, Advanced Fluid Dynamics, Prof. Hyoung Woo Oh (Ed.), ISBN: 978-953-51-0270-0, InTech, Available from:

http://www.intechopen.com/books/advanced-fluid-dynamics/turbulent-boundary-layer-models-theory-andapplications

\section{INTECH}

open science | open minds

\section{InTech Europe}

University Campus STeP Ri

Slavka Krautzeka 83/A

51000 Rijeka, Croatia

Phone: +385 (51) 770447

Fax: +385 (51) 686166

www.intechopen.com

\section{InTech China}

Unit 405, Office Block, Hotel Equatorial Shanghai

No.65, Yan An Road (West), Shanghai, 200040, China

中国上海市延安西路65号上海国际贵都大饭店办公楼405单元

Phone: +86-21-62489820

Fax: $+86-21-62489821$ 
(C) 2012 The Author(s). Licensee IntechOpen. This is an open access article distributed under the terms of the Creative Commons Attribution 3.0 License, which permits unrestricted use, distribution, and reproduction in any medium, provided the original work is properly cited. 